\title{
II.
}

\section{Ueber die Ausscheidung der Harnbestandtheile bei Fieberbewegungen.}

(Aus dem Laboratorium der medicin. Klinik des Prof. Dr. H. Eichhorst in Zürich.)

Von Dr. W. von Moraczewski.

Es gehört zu den bekanntesten Thatsachen, dass im Fieber die $\mathrm{N}$-Ausscheidung vermehrt ist, dagegen eine Retention von Chlor im Organismus stattfindet. Neben den Stickstoff sind die Phosphate und die Kalisalze zu stellen, neben das Chlor die Salze des Natriums. Die schönsten und besten Untersuchungen führten mit zur Feststellung dieser Befunde; so die Arbeiten von Traube und Jochmann ${ }^{1}$ ), Redtenbacher ${ }^{2}$ ), Ringer ${ }^{3}$, Brattler ${ }^{4}$ ), Haxthausen ${ }^{5}$ ), Rosenstein ${ }^{6}$ ), Riesenfeld ${ }^{7}$ ), Unruh ${ }^{3}$ ), Huppert ${ }^{9}$ ), Schleich ${ }^{10}$ ), E. Hallervorden ${ }^{11}$ ), Salkowsky ${ }^{12}$ ), Naunyn ${ }^{18}{ }^{14}$ ), Zulzer ${ }^{15}$ ), Röhmann ${ }^{16}$ ),

J) Traube u. Jochmann, Deutsche Klinik Bd. 46. S. 511. 1859.

2) Redtenbacher, Zeitschr. für rat. Medicin.

3) E. Ringer, Transact. of the med. chir. Soc. XLII. 1859. p. 361 .

4) Brattler, Archiv für path. Anat. und Phys. Bd.21. S. 456 .

5) Haxthausen, siehe Bd. 21 . S. 366.

6) Rosenstein, siehe Bd. 43 . S. 401.

7) Riesenfeld, siehe Bd. 47. S. 133.

8) Unrub, siehe Bd. 48. S. 228.

9) Huppert, Arch. für Heilkunde Bd. 7. S. 69., Bd. 10. S. 329. Bd. 10. S. 503.

10) Schleich, Archiv für exp. Path. und Pharm. Bd. 4. S. 42.

11) F. Hallervorden, siehe Bd. 12. S. 237.

$\left.{ }^{12}\right)$ Salkowsky, Archiv für pathol. Anat. und Phys. Bd. 48. S. 391.

13) Naunyn, Achiv für Anat. und Phys. Phys. Abt. 1870. S. 159.

14) Derselbe, Berl. Klin. Wochenschr. 1869. No. 4. S. 42.

15) Zülzer, Centr, für med. Wissensch. 1877. No. 42, 43.

16) Röhmann, Zeitschr. für klin. Med. Bd. I. S. 513. 
v. Limbeck ${ }^{1}$ ), Senator ${ }^{2}$ ), v. Terray ${ }^{3}$ ), Liebermeister ${ }^{4}$ ), Leyden ${ }^{5}$ ), S. Rem Pirei und v. Calcini ${ }^{6}$ ) und vielen anderen. Sowohl in den ältesten Arbeiten von Moos, Uhle, Ranke, Wasmuth, Bartels, wie in den oben citirten, die lange nicht vollständig erwähnt sind, werden meist die Regeln gebildet auf Grund längerer Beobachtung, einer Beobachtung, welche Tage betreffen, nicht Stunden (ausgenommen die Arbeit von Naunyn). Das Beobachtungsmaterial, welches fast alle Harnbestandtheile umfasste, lieferte somit eine Charakteristik des Fieberstadiums, weniger des Fieberanfangs und des Endes.

Erst die neuesten Beobachtungen, welche an dem "verkürzten" Fieber - Malaria - gemacht wurden, brachte Neues und Ueberraschendes mit sich. Auch der vorliegende Versuch wurde durch die Arbeit meines Collegen und Freundes Dr. Theodor Hitzig veranlasst. Das abnorme Verhalten der Chloride und des Phosphors bei Malaria, ein Verhalten, welches im schroffsten Widerspruch mit den Befunden des Stoffwechsels bei Fieber steht (v. Limbeck, v. Terray), lenkte meine Aufmerksamkeit auf die anderen Fiebertypen und brachte mich dazu, die alten Beobachtungen zu revidiren und selbständige Versuche anzustellen. - Es war allerdings auch den früheren Beobachtern nicht entgangen, dass nach dem Fieber der Stickstoff vermehrt ausgeschieden wird (Naunyn), oder dass die Kalisalzausscheidung nach dem Fieber abnorm sinke (Salkowsky) - oder dass das Ammoniak, welches im Fieber vermehrt ausgeschieden wird, nach dem Fieber dieses Verhalten in noch höherem Maasse zeige (Hallervorden). Auch die Retention von Chloriden wurde manchmal nach dem Fieber grösser (Ringer), ebenso die Vermehrung der Phosphorsäure (v. Haxthausen) im Harne.

Dieses also war bekannt und viel Neues kann unsere Arbeit nicht bringen. Sie soll vielmehr die Fiebertypen auf das Verhalten

1) v. Limbeci, Wien. Med. Wochenschrift. 1892.

$\left.{ }^{2}\right)$ Senator, Centr. für med. Wissensch. 1877. S. 389.

3) v. Terray, Zeitschr. für klin. Med. Bd. 21 . S. 514.

4) Liebermeister, Prog. Vierteljahr. 37. S. 63.

5) Leyden, Deutsch. Archiv für klin. Med. 1869. S.371.

6) S. Rem Picei e V. Calcini, H. Policlinico. 1894. April und Dezember. 
der Salze vor, während und nach dem Fieber untersuchen, die Resultate zusammenstellen und aus dem Gesichtspunkte betrachten, welche uns auf Grund früherer Beobachtung am naheliegendsten erscheint.

Das Material stammt aus der Klinik des Herrn Prof. Dr. Hermann Eichhorst, und ich bin ihm für das Entgegenkommen und stetiges Beistehen zur aufrichtigsten Dankbarkeit verpflichtet, ebenso dem Herrn Secundärarzt Dr. Th. Hitzig auf dessen Abtheilung die Kranken sich befanden und dessen freundliches Mitwirken viel zur Vollbringung dieser Arbeit beigetragen hat.

Die chemischen Methoden, welche ich hier benutzt habe, sind in meinen früheren Arbeiten oft beschrieben, und ich kann mich darauf beschränken, auf diese hinzuweisen (dieses Archiv Bd. 146, 150 u. s. w., Zeitschr. f. klinische Modic. Bd. 34, 35).

Die Kalium- und Natriumbestimmung geschah nach der bekannten Vorschrift von Fresenius: Glühen der $50 \mathrm{ccm}$ Harn mit Ammoniumsulfat behufs Umwandlung in schwer flüchtige Sulfatedes Kaliums und Natriums, Umsetzen des Glührückstandes mit Bariumchlorid und Bariumhydrat in Chloride des Natriums und Kaliums, Entfernen des überschüssigen Bariums durch Ammoniumcarbonat, Verjagen der überschüssigen Ammoniumsalze und Wiegen des Rükstandes im Platintiegel als Natriumchlorid und Kaliumchlorid.

Nachher wurde aus dem Rückstande das Kalium mit Platinchlorid gefällt, mit Alkohol und Aether ausgewaschen, jn siedendem Wasser gelöst, die Lösung abgedampft und der Abdampfrïckstand als Kaliumplatinchlorid gewogen.

Aus der gefundenen Kaliummenge wurde die Natriummenge ermittelt, indem man von der Summe der beiden Chloride das Kaliumchlorid subtrahirte.

Das Chlor warde nach Volhard, das Phosphor nach Neubauer, ebenso das Calcium, der Harnstoff nach Mörner, die Harnsäure nach Ludwig-Salkowsky, das Ammoniak nach Schlösing, die Alloxurbasen nach der von mir in diesem Archiv $\mathrm{Bd}$. 150 beschriebenen Methode bestimmt ${ }^{1}$ ).

Das Gleiche betrifft die Regelung der Diät und die Bestimmung der Nahrung. Die Analysen der Speisen, welche Herr Dr. Th.

1) Die neue Arbeit von Salkowsky in Pflüger's Archiv 1898 war damals noch nicht bekannt. 
Hitzig für seine Arbeit machte, bestätigten unsere früheren Untersuchungen und bilden genügende Garantie für die gleichmässige Herstellung der Kost, sowie der Zuverlässigkeit der Methoden.

Die Patienten waren unter sicherer Aufsicht und wurden von uns wo nöthig instruirt, damit die Untersuchung, welche peinlicher Vorsicht bedarf, vereinfacht werde. So war die Trennung des Harnes von dem Stuhl stets aufs Sorgfältigste durchgeführt, auch auf das pünktliche Entleeren der Blase Gewicht gelegt. Die Temperatur war durch geübte Wärterinnen gemessen.

Wir unterzogen unserer Beobachtung zwei Fälle von Febris continua mit einem allmählichen Absteigen und allmählichen Sinken der Temperatur (Typhus), einen Fall von Fieber, welcher mit Crise verläuft (Pneumonie), einen Fall von Malariafieber und einen von hektischem Fieber. - Die Untersuchung der anderen Fieber-Formen bei acuten Exanthemen, bei Gelenkrheumatismus u. s. w. haben wir unterlassen, da aus den früheren Beobachtungen zu ersehen war, dass solche Fälle weniger deutliche Resultate ergeben und individuellen Schwankungen unterworfen sind.

Um uns ein möglichst deutliches Bild zu schaffen, haben wir neben den Harnstoffbestimmungen die Bestimmung des Gesammtstickstoffs, der Harnsäure, der Xanthinbasen, des Ammoniaks ausgeführt. Ausserdem waren Chloride, Phosphate, Kalium, Natrium und Calcium bestimmt.

Bei der Pneumonie wurde der Harn bis zur Crise und nach der Crise gesammelt, bei Malariafieber in fünf Portionen, welche dem Stadium $1^{0}$ des Schüttelfrostes, $2^{0}$ des Fiebermaximums, $3^{0}$ der Fieberremission, $4^{0}$ der weiteren Fieberabnahme, $5^{0}$ der Fieberlosigkeit entsprachen. Bei Febris hectica in vier Portionen dem Stadium $1^{0}$ des beginnenden Fiebers, $2^{0}$ der Fieberhöhe, $3^{0}$ der Fieberremission, $4^{0}$ der Fieberlosigkeit entsprechend. Bei 'Typhus I waren in drei Perioden: $1^{0}$ der Fieberhöhe $2^{\circ}$ des Fieberabfalls, $3^{0}$ der fieberlosen Zeit der Harn als Tages- und Nachtharn gesammelt. In Typhus II wurde die ganze Portion von 24 Stunden untersucht, dafür ohne Unterbrechung während des ganzen Verlaufes, welcher die Periode $1^{0}$ des Ansteigens, $2^{0}$ des continuirlichen Fiebers, $3^{0}$ des Sinkens des Fiebers betroffen. Näheres wird bei jedem einzelnen Falle besprochen.

Die Zusammenstellung der Tabellen war verschieden je nach 
den Fällen. So wurden bei Malaria- und hektischem Fieber die stündlichen Mengen hervorgehoben; dagegen die tägliche Bilanz weniger betont, da der Koth nicht in Rechnung gezogen werden konnte. Bei den Fällen von Typhas and Pneumonie wurde neben den stündlichen Mengen auch die Bilanz genan festgestellt. Da, wo die Bestimmung der stündlichen Mengen ohne Werth erschien, wurde sie aus den Tabellen weggelassen.

Die hier angeführten Fälle sind als Durchschnittfälle wiederholter Beobachtungen za betrachten, welche wir in der Klinik gesammelt haben und deren Anführung zur Controle die Arbeit unnütz ausdehnen würde.

Fall I. B. 0., 17 Jahr. Typhus abdominalis.

Anamnese: Patient stammt aus gesunder Familie. Als Kind machte er die Masern durch, will nachher stets gesund gewesen sein. Vor 10 Tagen erkrankte er unter den Erscheinungen von Kopfschmerzen, AppetitIosigkeit und Durchfall.

St. praesens. An den äusseren und inneren Organen nichts Abnormes zu entdecken. Roseola spärlich, Miilz mässig vergrössert.

Der Patient wurde auf Diät gesetzt, welche aus $1200 \mathrm{ccm}$ Milch, $800 \mathrm{ccm}$ Bouillon mit Ei, 500-800 $\mathrm{cm}$ Wein bestand.

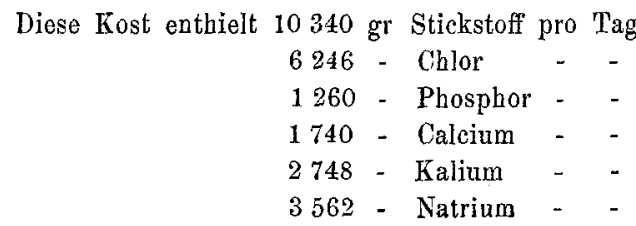

und wurde wäbrend der ganzen Versuchsreibe beibehalten.

Der Koth wurde nicht in Rechnung gebracht, da die Tage der Untersuchung herausgegriffen waren aus der Mitte der einzelnen Perioden. Die Nicbtbestimmung des Koths bringt Fehler mit sich, welche das Calcium allein treffen. Die Menge der anderen Bestandtheile wird durch die Kothausscheidung wenig geändert.

Der Harn wurde in Tag- und Nachtportionen gesammelt an drei Tagen.

Der erste Tag stammt aus der Fieberperiode.

$\begin{array}{rcccc}\text { Temperatur während } 24 \text { Stunden } & 38.6 & 39.5 & 38.1 & 38.5 \\ \text { Pulszahl } & 106 & 108 & 104 & 100\end{array}$

Der Kranke war einmal am Tage gebadet, die Temperatur des Wassers war etwa $30^{\circ}$.

Der zweite Tag der Harnuntersuchung war während des Stadiums der abnehmenden Temperatur ausgesucht.

$\begin{array}{lllll}\text { Temperatur am Tage der Untersuchung } & 37.2 & 36.8 & 38.2 & 38.1\end{array}$

$\begin{array}{lllll}\text { Pulszabl } & 76 & 80 & 92 & 88\end{array}$


Der dritte Tag stammt aus der fieberfreien Zeit.

$\begin{array}{rllll}\text { Temperatur } & 36.4 & 36.6 & 36.0 & 36.5 \\ \text { Pulszahl } & 76 & 68 & 68 & 76\end{array}$

Diese Untersuchung sollte den Typus des Fieberharnes und des Harnes der fieberfreien Perioden zeigen. Die gefundenen Zahlen waren auf stündliche Ausscheidung berechnet.

Die Bilanz wurde obne Berücksichtigung des Kothes gemacht.

Tabelle I. B. 0., 17 Jahr. Typus abdominalis.

$\begin{array}{rcccc}\text { Temperatur } & 38.6 & 39.5 & 38.1 & 38.5 \text { Fieberböhe. } \\ \text { Puls } & 106 & 108 & 104 & 100\end{array}$

Von 6 Uhr Morgens bis 6 Thr Abends.

Harnmenge $1300 \mathrm{ccm}$ Sp. Gewicht 1015.

N 1.001 pCt.

pro $12 \mathrm{~h}^{\prime} 13.013$

pro Stunde 1.084

Cl 0.212 -

2.756

0.229

P 0.077 -

1.141

0.095

$\mathrm{Ca} 0.0082$

0.106

0.009

K 0.159

2.067

0.172

Na 0.149

1.937

0.161

Harnst. 0.840

Harns. 0.014

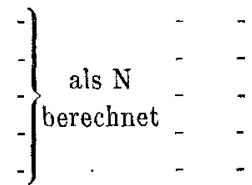

10.920

0.910

Alloxb. 0.018

0.182

0.015

Xanthinb. 0.004

NHs 0.057

0.234

0.019

0.054

0.064

Von $6 \mathrm{Uhr}$ Abends bis $6 \mathrm{Uhr}$ Morgens.

Harnmenge $590 \mathrm{ccm}$., Sp. Ger. 1016.

N 1.218 pCt.

Cl 0.206 -

P 0.1177 -

Ca 0.0058

K 0.183

Na 0.116

Harnst. 0.984

Harns. 0.012

Alloxb. 0.021

Xanthinb. 0.009

NHs 0.079

Nahrung

N 10.340

C] 6.246

P 1.260

Ca 1.740

K $\quad 2.748$

$\mathrm{Na} \quad 3.562$ pro 12 Std. 7.186

- $\quad-1.215$

- $\quad-0.694$

- $\quad 0.034$

- $\quad-\quad 0.079$

- $\quad$ - 0.684

$\begin{array}{llll} & - & - & 5.806 \\ \text { als N } & - & - & 0.0708\end{array}$

0.177

0.0531

0.466

Resultat:

Harn

20.199

3.971

1.835

0.140

3.146

2.621 pro Stunde $0.599 \mathrm{gr}$.

0.101 -

0.058 -

$0.003-$

0.090 -

0.057 -

0.484 -

0.006 -

$0.015-$

$0.004-$

0.040 -

Bilanz

$-9.859 \mathrm{~g} \mathrm{~N}$

$+2.275-\mathrm{Cl}$

$-0.575-\mathrm{P}$

$+1.600-\mathrm{Ca}$

$-0.398 \cdot \mathrm{K}$

$+0.941-\mathrm{Na}$ 


\subsection{Harnstoff $\mathrm{N}$ \\ 0.253 Harnsäure $\mathrm{N}$ \\ 0.411 Alloxb. N \\ 0.158 Xanthinb. $\mathrm{N}$ \\ 1.208 Ammoniak $\mathrm{N}$}

Anm. Rechnet man die eventuellen Verluste im Koth mit, so wird das Resultat noch verschärft, nicht wesentlich geändert. $\mathrm{N}$ kann noch mehr ausgeschieden werden, ebenso $\mathrm{P}$, Calcium kann weniger retinirt werden. Chlor, Kalium und Natrium bleiben ohne wesentliche Aenderung, da die Menge im Koth sebr gering ist.

Fieberabfall.

\begin{tabular}{|c|c|c|c|}
\hline Temperatur & 37.2 & 36.8 & 38.2 \\
\hline Pulszahl & 76 & 80 & 92 \\
\hline
\end{tabular}

Von 6 Uhr Morgens bis $6 \mathrm{Uhr}$ Abends.

Harnmenge $510 \mathrm{ccm}$, spez. Gewicht 1013.

\begin{tabular}{|c|c|c|c|c|c|}
\hline $\mathrm{N} 0.7945$ & pOt. & 2 Stunden & 4.052 & pro Stunde & 0.338 \\
\hline Cl 0.2548 & - & - & 1.299 & - & 0.108 \\
\hline P 0.0594 & - & - & 0.3029 & - & 0.025 \\
\hline $\mathrm{Ca} 0.0055$ & - & - & 0.0281 & - & 0.002 \\
\hline $\mathrm{K}^{\prime} 0.1170$ & - & - & 0.5967 & - & 0.049 \\
\hline $\mathrm{Na} 0.167$ & - & - & 0.8517 & - & 0.071 \\
\hline toff 0.672 & - & - & 3.427 & - & 0.289 \\
\hline ure 0.0098 & - & - & 0.0499 & - & 0.004 \\
\hline $\mathrm{xb} .0 .0126$ & - & - & 0.0643 & - & 0.005 \\
\hline nb. 0.0023 & - & - & 0.0144 & - & 0.001 \\
\hline iak 0.0434 & - & - & 0.2213 & - & 0.0188 \\
\hline
\end{tabular}

Von 6 Uhr Abends bis 6 Uhr Morgens.

Harnmenge $380 \mathrm{ccm}$, spec. Gewicht 1020.

\begin{tabular}{|c|c|c|c|c|c|c|}
\hline $\mathrm{N}$ & 1.330 & pCt. pro & 12 Stunden & 5.504 & pro Stunde & 0.421 \\
\hline $\mathrm{Cl}$ & 0.309 & - & - & 1.295 & - & 0.108 \\
\hline $\mathrm{P}$ & 0.1566 & - & - & 0.710 & - & 0.060 \\
\hline $\mathrm{Ca}$ & 0.0075 & - & - & 0.0285 & - & 0.002 \\
\hline K & 0.1877 & - & - & 0.713 & - & 0.060 \\
\hline $\mathrm{Na}$ & 0.2126 & - & - & 0.806 & - & 0.065 \\
\hline Harnstoff & 1.176 & - & - & 4.474 & - & 0.373 \\
\hline Harnsäure & 0.017 & - & - & 0.0646 & - & 0.005 \\
\hline Alloxb. & 0.0224 & - & - & 0.851 & - & 0.007 \\
\hline Xanthinb. & 0.0054 & - & - & 0.0205 & - & 0.002 \\
\hline NHs. & 0.0616 & - & - & 0.2341 & - & 0.020 \\
\hline \multicolumn{3}{|c|}{ Nabrung } & \multicolumn{2}{|l|}{ Harn } & \multicolumn{2}{|l|}{ Resultat } \\
\hline \multicolumn{3}{|c|}{ N 10.349} & \multicolumn{2}{|l|}{9.106} & \multicolumn{2}{|c|}{+1.234 oder - } \\
\hline \multicolumn{3}{|c|}{ Cl. $\quad 6.246$} & \multicolumn{2}{|l|}{2.594} & \multicolumn{2}{|c|}{+3.652} \\
\hline \multicolumn{3}{|c|}{$\mathrm{P} \quad 1.260$} & \multicolumn{2}{|l|}{1.013} & \multicolumn{2}{|c|}{+0.247 oder -} \\
\hline \multicolumn{3}{|c|}{$\mathrm{Ca} \quad 1.740$} & \multicolumn{2}{|l|}{0.056} & \multicolumn{2}{|c|}{+1.684} \\
\hline
\end{tabular}




\begin{tabular}{rrrr}
\multicolumn{2}{c}{ Nahrung } & Harn & Resultat \\
$\mathrm{K}$ & 2.748 & 1.319 & +1.429 \\
$\mathrm{Na}$ & 3.562 & 1.657 & +1.905
\end{tabular}

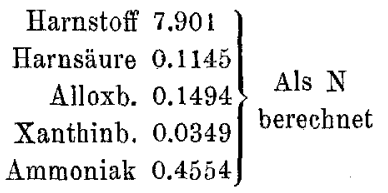

A n m. Durch die Berücksichtigung der Verluste im Koth können die Resultate wesentlich geändert sein, so kann ein $N$ nach Phospborverlust entstehen; das Calcium kann weniger retinirt werden. Die anderen Grössen bleiben bestehen. Hervorzuheben ist das Verbalten von $\mathrm{K}$, welches - in Uebereinstimmung mit der Beobachtung von Salkowsky - sparsam ausgeschieden wird.

\section{Fieberfrei.}

\begin{tabular}{|c|c|c|c|c|}
\hline Temperatur & 36.4 & 36.6 & 36.0 & 36.5 \\
\hline Pulszahl & 76 & 68 & 68 & 76 \\
\hline
\end{tabular}

Harumenge $1200 \mathrm{ccm}$, spec. Gewicht 1013.

$\begin{array}{rlcllll}\text { N } 0.7945 & \text { pCt. } & \text { pro 12 Stunden } 9.534 & \text { pro Stunde } 0.794 \\ \text { Cl } 0.3944 & - & - & 4.733 & - & 0.394 \\ \text { P } 0.0454 & - & - & 0.545 & - & 0.045 \\ \text { Ca } 0.0090 & - & - & 0.108 & - & 0.009 \\ \text { K } 0.1990 & - & - & 2.388 & - & 0.1990 \\ \text { Na } 0.2040 & - & - & 2.448 & - & 0.2040 \\ \text { toff } 0.680 & - & - & 8.160 & - & 0.680 \\ \text { ure } 0.0090 & - & - & 0.108 & - & 0.009 \\ \text { xb. } 9.0136 & - & - & 0.163 & - & 0.0136 \\ \text { nb. } 0.0046 & - & - & 0.053 & - & 0.0046 \\ \text { NHs } 0.0420 & - & - & 0.504 & - & 0.042\end{array}$

Von 6 Uhr Abends bis 6 Ubr Morgens.

$\begin{array}{rrrrrrr}\text { N } 1.155 & \text { pCt. } & \text { pro 12 Stunden } 6.468 & \text { pro Stunde } 0.539 \\ \text { Cl } 0.4368 & - & - & 2.445 & - & 0.204 \\ \text { P } 0.0864 & - & - & 0.484 & - & 0.041 \\ \text { Ca } 0.0664 & - & - & 0.0358 & - & 0.003 \\ \text { K } 0.297 & - & - & 1.663 & - & 0.139 \\ \text { Na } 0.209 & - & - & 1.171 & - & 0.099 \\ \text { Harnstoff } 0.966 & - & - & 5.409 & - & 0.451 \\ \text { Harnsäure } 0.014 & - & - & 0.0784 & - & 0.006 \\ \text { Alloxb. } 0.0189 & - & - & 0.1058 & - & 0.009 \\ \text { Xanthinb. } 0.0049 & - & - & 0.0274 & - & 0.003 \\ \text { NHs } 0.0616 & - & - & 0.3449 & - & 0.029 \\ \text { Nahrung } & \text { Harn } & & \text { Resultat } & \\ \text { N } 10.340 & 16.002 & & -5.662 & \\ \text { Cl } 6.246 & 7.178 & & -0.932 & \\ \text { P } 1.260 & 1.029 & & +0.231 & \end{array}$




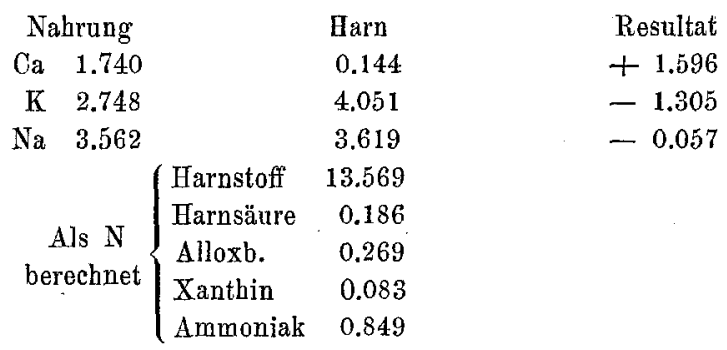

Anm. Das Resultat kann durch die Berücksichtigung der Kothbestandtheile nicht wesentlich geändert werden. Stickstoff wäre vermehrt ausgeschieden, Phosphor vielleicht im Gleichgewicht, Calcium weniger retinirt. Die anderen Bestandtheile wurden alle noch vermehrt ausgeschieden. Hier wiederum das Verhalten des $\mathrm{K}$ auffallend, in dem seine Ausscheidung deutlich vermehrt ist.

Dieser Fall sollte uns ein Bild über das Verhalten der Salze im Fieber verschaffen. Stellt man die Resultate des ersten Tages (Fieberhöhe) mit denen des letzten zusammen, so ist das Bild ein recht typisches. Dort die Retention von Chlor und Natrium, der Verlust von Phosphor; hier der Verlust von Chlor und Natrium und die Retention von Phosphor - also gerade umgekehrt. Das Verhalten des Stickstoffes und der Kalisalze soll uns hier nicht irre führen. Es unterliegt bekanntlich anderen Gesetzen, deren Natur wir zu besprechen haben. - Die Periode des Fieberabfalles zeigt einen regelrechten Uebergang zwischen den beiden Perioden, wenn man die Correctur anbringt, welche die Nichtberechnung der Stuhlbestandtheile erfordert.

Was die stickstoffhaltigen Bestandtheile des Harnes angeht, so waren auch diese ihrem Verhalten nach recht typisch: während des Fiebers vermehrte Harnsäure, Xanthinbasen, Alloxurbasen, überhaupt (als Summe von Xanthinbasen und Harnsäure berechnet) vermehrte Ammoniakausscheidung.

Fieber $\frac{\text { Harnst. }}{\mathrm{N}} 0.83 \quad \frac{\text { Harns. }}{\mathrm{N}} 0.012 \quad \frac{\text { Alloxb. }}{\mathrm{N}} 0.020 \quad \frac{\mathrm{NHs}}{\mathrm{N}} 0.060$ Fieberlose Ber. $\quad-\quad 0.85 \quad-0.011 \quad-0.017 \quad-0.053$

Auch das von $Z$ ülzer eingeführte Verhältniss $\frac{P}{N}$ gestaltete sich typisch. Fieber $\frac{P}{N} 0.0917 \quad$ fieberfreies Stadium $\frac{P}{N} 0.064$

Bildet man schliesslich das Verhältniss des $\mathrm{N}$ in den 
anderen Bestandtheilen in Nahrung und Harn, so ergiebt sich für das Stadium des Fiebers:
$\mathrm{N}: \mathrm{Cl}: \quad \mathrm{P}$
$\mathrm{Ca}$ :
$\mathrm{K}: \mathrm{Na}$ :
$1: 0.6$
0.12
$0.17 \quad 0.27$
0.35 in der Nahrung
$1: 0.198$
$0.0917\left(0.007^{1}\right) 0.157$
0.131 in der Ausscheidung.

Für die Zeit der Fieberabnahme:

$\begin{array}{cccccc}\mathrm{N}: & \mathrm{Cl}: & \mathrm{P}: & \mathrm{Ca}: & \mathrm{K}: & \mathrm{Na}: \\ 1: & 0.286 & 0.111 & 0.006 & 0.146 & 0.182\end{array}$

Für die fieberfreie Zeit:

$$
\begin{array}{cccccc}
\mathrm{N}: \mathrm{Cl}: & \mathrm{P}: & \mathrm{Ca}: & \mathrm{K}: & \mathrm{Na} & \\
1: 0.62 & 0.12 & 0.17 & 0.27 & 0.35 \text { in der Nabrung } \\
1: 0.448 & 0.064 & (0.009) & 0.253 & 0.226 & \text { in der Ausschejdung. }
\end{array}
$$

$\mathrm{N}$ : Harnst. Harns. Alloxb. NHs

$\begin{array}{lllll}\text { Fieberhöhe } & 0.836 & 0.0126 & 0.0205 & 0.0604 \\ \text { Fieberabnahme } & 0.868 & 0.0125 & 0.0164 & 0.0500 \\ \text { Fieberfreie Zeit } & 0.878 & 0.0084 & 0.0168 & 0.0530\end{array}$

Man ersieht daraus: 1. dass keiner der Harnbestandtheile so reichlich ausgeschieden wird, wie die Stickstoffverbindungen, 2. dass die fieberfreie Periode sich durch die Aufbesserung des Verhältnisses der Chloride und der Kalisalze auszeichnet.

Soweit also das typische Verhalten im Fieber. Im Falle II, welcher auch ein Typhusfall ist, werden wir die Aenderungen im Verbältniss zur Steigerung der Körperwärme studiren können.

Fall Ir. Z. A., 32 Jahre. Typhus abdominalis.

Anamnese ergiebt nichts, was von Belang wäre. Patient stammt aus gesunder Familie, war bis jetzt völlig gesund. Das jetzige Leiden begann seit 12 Tagen. Patient verlor Schlaf und Appetit, füblte sich unwoh] nnd liess sich behufs besserer Verpflegung in das Krankenbaus aufnehmen.

St. praesens. Die inneren und äusseren Organe bieten keine wesentliche Veränderung. Ausser einer deutlichen Milzschwellung ist nichts zu entdecken.

Der Patient erhielt die gleiche Diät, wie Fall I $1200 \mathrm{ccm} \mathrm{Milch,} 800$ Bouillon mit Ei und $\frac{1}{2}$ Ltr. Wein. Diese Nahrung blieb bis zum Ende des Versuches unverändert. Die Menge wurde wie in allen übrigen Fällen durch Maass bestimmt. Alle Nahrungsmittel wurden von uns selbst analysirt.

Der Patient wurde von uns auf die in Betracht kommenden Bestand. theile untersucht und ihre Menge pro Tag in Rechnung gebracht, indem aus den Procenten die Gesammtmenge berechnet wurde und diese in die Zabl der Tage dividirt.

Der Harn wurde täglich gemessen und täglich untersucht, daraus der tägliche Verlust an $\mathrm{N}, \mathrm{Cl}, \mathrm{P}, \mathrm{Ca}, \mathrm{K}, \mathrm{Na}$, NHs und anderen Stickstoffverbindungen bestimmt. Alle Zahlen wurden als $\mathrm{Cl}, \mathrm{Na}, \mathrm{K}, \mathrm{P}, \mathrm{Ca}$ berechnet

1) Nur im Harne. 
(nicht als $\mathrm{P}_{2} \mathrm{O}_{5}, \mathrm{NaCl}$ u. s. w.). Die Stickstoffverbindungen wurden alle auf Stickstoff umgerechnet, somit die Menge des Stickstoffs angegeben, welche als NHs, Harnsäure, Alloxurbasen (Harnsäure und Xanthin), Xantbin ausgeschieden wurde.

Die Temperatur war im Anfang des Versuches im Steigen begriffen, erreichte ihr Maximum, fiel dann bis zur Norm zurück. Somit war der Einfluss des Steigens und des Sinkens beobachtet.

\begin{tabular}{|c|c|c|c|c|c|}
\hline \multirow[t]{2}{*}{ 13. Juli } & 38.9 & 38.7 & 39.5 & 39.2 & Temp. \\
\hline & 88 & 90 & 100 & 96 & Pulszahl \\
\hline \multirow[t]{2}{*}{ 14. Juli } & 39.0 & 39.5 & 39.0 & 38.6 & Temp. \\
\hline & 92 & 100 & 96 & 92 & Pulszah \\
\hline \multirow[t]{2}{*}{ 15. Juli } & 37.8 & 38.4 & 38.5 & 39.0 & Temp. \\
\hline & 100 & 96 & 100 & 92 & Pulszahl \\
\hline \multirow[t]{2}{*}{ 16. Juli } & 38.5 & 39.5 & 39.9 & 39.6 & Temp. \\
\hline & 92 & 92 & 96 & 96 & Pulszahl \\
\hline \multirow[t]{2}{*}{ 17. Juli } & 38.6 & 39.6 & 38.8 & 37.5 & Temp. \\
\hline & 89 & 92 & 96 & 84 & Pulszahl \\
\hline \multirow[t]{2}{*}{ 18. Juli } & 37.5 & 38.1 & 39.5 & 39.0 & Temp. \\
\hline & 84 & 96 & 100 & 92 & Pulszahl \\
\hline \multirow[t]{2}{*}{ 19. Juli } & 37.6 & 39.4 & 40.1 & 39.0 & Temp. \\
\hline & 92 & 96 & 100 & 101 & Pulszahl \\
\hline \multirow[t]{2}{*}{ 20. Juli } & 37.6 & 37.2 & 37.2 & 38.2 & Temp. \\
\hline & 100 & 100 & 96 & 100 & Pulszahl \\
\hline \multirow[t]{2}{*}{ 21. Juli } & 36.5 & 37.2 & 39.3 & 38.9 & Temp. \\
\hline & 96 & 96 & 96 & 112 & Pulszahl \\
\hline \multirow[t]{2}{*}{ 22. Juli } & 38.9 & 37.5 & 37.8 & 38.5 & Temp. \\
\hline & 104 & 101 & 101 & 88 & Pulszahl \\
\hline \multirow[t]{2}{*}{ 23. Juli } & 37.0 & 37.1 & 38.3 & 38.5 & Temp. \\
\hline & 92 & 88 & 100 & 96 & Pulszahl \\
\hline \multirow[t]{2}{*}{ 24. Juli } & 36.2 & 37.3 & 38.2 & 38.3 & Temp. \\
\hline & 84 & 98 & 100 & 92 & Pulszahl \\
\hline
\end{tabular}

Tabelle II. Typhus abdominalis.

14. Juli. Temp. 39.1. Harnmenge $800 \mathrm{ccm}$. Spec. Gew. 1026.

N 2.289 pCt. 18.312 pro Tag 0.5267 i. Koth 10.340 i. d. Nahr. -8.499

Cl $0.2427-1.9416-0.4669-6.246-13.837$

$\mathrm{P} 0.1450-1.1600-0.2912-1.260-0.191$

$\mathrm{Ca} 0.0175-0.1400-0.1901-1.740-+1.310$

$\mathrm{K} 0.0985-0.7880-0.3795-2.748-+1.581$

$\mathrm{Na} 0.0905-0.7240-0.1683-3.562-+2.670$

Harnst: $1.806-14.4480$

Harns. $0.0224-0.1792 \quad-$

Alloxb. $0.0289-0.2312 \quad-$

Xanth. $0.0065-0.0520-$

NHs $0.09035,0.7224$ - 
15. Juli. Temp. 38.4. Harnmenge $820 \mathrm{ccm}$. Spec. Gew. 1027.

N 1.757 pCt. 14.507 proTag 0.5267 i. Koth 10.340 i. d. Nahr. -4.694 .

$\mathrm{Cl} 0.4371-3.496-0.4669-6.246-1.283$

$\mathrm{P} 0.1250-1.025-0.2912-1.260-0.056$

Ca $0.0222-0.1810-0.1901-1.740-+1.369$

K $0.0802-0.6576-0.3795-2.748-+1.711$

$\mathrm{Na} 0.1495-1.2259-0.1683-3.562-+2.168$

Harnst. $1.526 \quad-12.5132 \quad-$

Harns. $0.0217-0.1779 \quad-$

Alloxb. $0.0289-0.2379$ -

Xanth. $0.0072-0.0600$ -

NHs $0.0861-0.7860 \quad-$

16. Juli. Temp. 39.4. Harnmenge $1220 \mathrm{ccm}$. Spec. Gew. 1026 .

N 1.6625 pCt. 20.2775 pro Tag 0.5267 i. Koth 10.340 i. d. Nahr. -10.465

Cl $0.4368-5.3289-0.4669-6.246-\quad+0.451$

$\mathrm{P} 0.0860-1.0492-0.2912-1.260-0.080$

$\mathrm{Ca} 0.0076-0.0927-0.1901-1.740-\quad+1.457$

K 0.0832 pCt. 0.0150 pro Tag 0.3795 im Koth 2.748 i. d. Nahr. + 1.353

$\mathrm{Na} 0.1493-1.8215-0.1683-3.562-+1.572$

Harnst. $1.518-19.519$ -

Harns. $0.0210-0.2562 \quad-$

Alloxb. 0.0298 - 0.3635 -

Xanth. $0.0088-0.1073$ -

17. Juli. Temp. 38.6. Harnmenge $700 \mathrm{ccm}$. Spec. Gew. 1030.

N 1.967 pCt. 13.769 pro die 0.5267 i. Koth 10.340 i. d. Nahr. -3.956

Cl $0.2123-1.486-0.4669-6.246-+4.292$

$\mathrm{P} 0.1520-1.064-0.2912-1.260-0.095$

$\mathrm{Ca} 0.0191-0.1337-0.1901-1.740-+1,419$

K $0.1134-0.7938-0.3795-2.748-+1.575$

Na $0.0664-0.4648-0.1683-3.562 \quad-\quad+2.929$

Harnst. $1.736-12.1520 \quad-$

Harns. 0.02345 - 0.1641 -

Alloxb. 0.03174 - 0.2221 .

Xanth. $0.00829-0.0580$ -

NHs $0.0972-0.6804 \quad-$

18. Juli. Temp. 38.5. Harnmenge $800 \mathrm{ccm}$. Spec. Gewicht 1026 .

N 2.037 pCt. 16.296 pro die 0.5267 i. Koth 10.340 i. d. Nahr. -6.483

Cl $0.3034-2.427-0.4669-6.246-+3.353$

$\mathrm{P} 0.1890-1.513-0.2912-1.260-0.544$

Ca $0.0288-0.2300-0.1901-1.740-+1.320$

$\mathrm{K} 0.0893-0.7144-0.3795-2.748-+1.654$

$\mathrm{Na} 0.0785-0.6280-0.1683-3.562-+2.766$ 
Harnst. 1.708 pCt. 13.6640 pro die

Harns. $0.0297-0.2380$
Alloxb. $0.0389-$
Xanth. $0.0092 \div 0.3115 \quad-$
NHs $0.1127-0.0735$ -

19. Juli. Temp. 39.0. Harnmenge $500 \mathrm{ccm}$. Spec. Gewicht 1030.

N 1.9915 pCt. 9.9575 pro die 0.5267 i. Koth 10.340 i. d. Nabr. -0.144

$\mathrm{Cl} 0.3034-1.517-0.4669-6.246-6.263$

$\begin{array}{llllllll}\mathbf{P} 0.1425 & -0.713-0.2912 & - & -1.260- & - & +0.257\end{array}$

$\mathrm{Ca} 0.01793-0.0897-0.1901-1.740-0.37951$

$\mathrm{K} 0.1125-0.5625-0.3795-2.748 \quad-\quad+1.806$

$\mathrm{Na} 0.0459-0.2295-0.1683-3.562-0.165$

Harnst. $1.708-8.5400 \quad-$

Harns. $0.02625-0.1313-$

Alloxb. $0.03197-0.1598$

Xanth. $0.00572-0.0285$

NHs $0.1519 \quad-0.7595$

20. Juli. Temp. 37.5. Harnmenge $450 \mathrm{ccm}$. Spec. Gewicht 1028.

N 2.597 pCt. 11.687 pro die 0.5267 i. Koth 10.340 i. d. Nahr. -1.884

Cl $0.2548-1.147-0.4669-6.246-4.632$

$\mathrm{P} 0.1640-0.738-0.2912-1.260-\quad+0.231$

$\mathrm{Ca} 0.0262-0.1180-0.1901-1.740 \quad-\quad+1.432$

$\mathrm{K} 0.0435-0.1958-0.3795-2.748 \quad-\quad+2.173$

$\mathrm{Na} 0.0458-0.2061-0.1683-3.562-\quad+3.188$

Harnst. $1.736 \quad-7.812 \quad-$

Harns. $0.0290-0.1305-$

Alloxb. $0.0364-0.1639$ -

Xanth. $0.0074-0.0334-$

NHs $0.1820-0.8190 \quad-$

21. Juli. Temp. 38.0. Harnmenge $600 \mathrm{ccm}$. Spec. Gewicht 1026.

$\mathrm{N} 2.072$ pCt. 12.439 pro die 0.5267 i. Koth 10.340 i. d. Nahr. -2.618

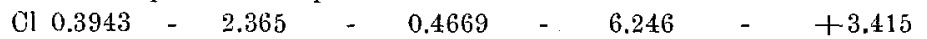

$\mathrm{P} 0.1250-0.750-0.2912-1.260-1.740-10.229$

$\mathrm{Ca} 0.0155-0.093-0.1901-1.740-1.463$

$\mathrm{K} 0.0825-0.4950-0.3795-2.748-0.1 .873$

$\mathrm{Na} 0.0710-0.4260-0.1683-3.562-0.912-+2.868$

Harnst. $1.652 \quad-\quad 9.912 \quad-$

Harns. $0.0329-0.1974 \quad-$

Alloxb. $0.0409-0.2457$

Xanth. $0.0080-0.0483-$

NHs $0.1400-0.8400 \quad-$

22. Juli. Temp. 38.1. Harnmenge $600 \mathrm{cem}$. Spec. Gewicht 1027.

$\mathrm{N} 1.7500 \mathrm{pCt} .10 .500$ pro die 0.5267 i. Koth 10.340 i. d. Nahr. -0.686

Cl $0.3701-2.220-0.4669-6.246-+3.260$ 


$\begin{array}{rlllllllll}\text { P } 0.1150 & \text { pCt. } & 0.690 & \text { pro die } & 0.2912 & \text { i. Koth } & 1.260 & \text { i.d. Nahr. }+0.279 \\ \text { Ca } 0.0164 & - & 0.0984 & - & 0.1901 & - & 1.740 & - & +1.458 \\ \text { K } 0.0810 & - & 0.486 & - & 0.3795 & - & 2.748 & - & +1.883 \\ \mathrm{Na} 0.0923 & - & 0.5538 & - & 0.1683 & - & 3.562 & - & +2.840 \\ \text { Harnst. } 1.680 & - & 9.080 & - & & & & & \\ \text { Harns. } 0.0294 & - & 0.1764 & - & & & & & \\ \text { Alloxb. } 0.0373 & - & 0.2235 & - & & & & & \\ \text { Xanth. } 0.0078 & - & 0.0471 & - & & & & & \\ \text { NHs } 0.1232 & - & 0.7392 & - & & & & \end{array}$

Bei Betrachtung der zweiten Tabelle fällt es vor allem auf, dass der Organismus anders zu reagiren scheint auf die Erhöhung der Temperatur im Anfangstadium als in dem Stadium des Fieberabfalls. So ist das Ansteigen der Temperatur in den ersten Tagen mit einer Mehrausscheidung des Stickstoffs verbunden. Das Sinken der Temperatur bewirkt ein Sinken der Stickstoffmenge. Ist aber einmal die Temperatur zur Norm gesunken, so wird eine Erhöhung derselben von einer Verminderung der N-Ausscheidung begleitet. (Diese Regel ist aber durchaus nicht immer zutreffend, und wir werden besonders die Fälle hervorheben, wo sie zur Geltung kommt. Die meisten Beobachtungen ergeben einen Parallelismus zwischen Fieberhöhe und Stickstoffverlust.)

Die Chlorausscheidung sollte dem Stickstoff antagonistisch verlaufen, d. h. mit der Erhöhung der Temperatur sinken und mit dem Fallen der Körperwärme steigen. In unserem Fall trifft dieses meistens zu; aber auch hier ist eine Steigerung der Temperatur nach dem Fieberanfall mit einer Steigerung der Chlorausscheidung verbunden, eine Beobachtung, die von vielen Autoren gemacht (Rosenstein, Brattler) aber nicht genügend betont wurde.

Die Phosphorausscheidnng zeigt eine gewisse Trägheit: sie überdauert das Sinken der Temperatur and sinkt erst dann, wenn das Fieber längst vorbei ist. Sinkt die Temperatur, so kann die Phosphorausscheidung steigen; steigt jene, so kann diese fallen, und solches Verhalten finden wir in unserem Fall neben den oben genannten Abnormitäten der Stickstoff- und Chlorausscheidung.

Die Ausscheidung von Calciumsalzen wurde bis jetzt wenig beobachtet, man hat somit keine Anhaltspunkte und muss sich auf die blosse Hinstellung dieser Beobachtung beschränken. In 
diesem Falle verhielt sich die Calciumausscheidung der Temperatur antanogistisch. Jedes Sinken bewirkte ein Steigen der Calciumausfuhr, jedes Steigen ein Sinken derselben. $O b$ dies speciell in Typhus der Fall ist, kann man nicht entscheiden.

Die Kalisalze sind laut der Untersuchung von Salkowsky während des Fiebers vermehrt ausgeschieden, sinken dann nach dem Fieber sehr bedeutend und heben sich ganz allmählich. Wir können der Regel vollkommen beistimmen. Es scheint sogar, dass dieses Bild von dem zufälligen Sinken und Steigen der Temperatur nicht beeinflusst wird. - Es ist zwar bei raschem Ansteigen der Temperatur ein Sinken der Kalisalzausscheidung beobachtet, bei Sinken der Temperatur ein Ansteigen; aber dadurch ist die Geltung der Regel nicht geschmälert. Der Parallelismus, welchen man $z$ wischen $P$ und $\mathrm{K}$ zu erblicken glaubte, existirt in diesem Falle nicht; oft ist ein divergirendes Verhalten notirt.

Die Natronsalze verhalten sich genau wie das Chlor, zeigen also in unserem Falle die gleichen Schwankungen und Abnormitäten, wie sie bei Besprechung der Chlorausscheidung hervorgehoben warden.

Die Ausscheidung des Ammoniaks bedarf keiner besonderen Besprechung. Nach Hallervorden (a.a. 0.) soll seine Ausscheidung im Fieber vermehrt sein, somit wäre es dem Phosphor und N. an die Seite zu stellen. In unseren Fällen geht wirklich die NH's-Ausscheidung der des Phosphors parallel und zeigt mit ihr die beim Phosphor besprochenen Schwankungen. Eine Parallele zwischen $\mathrm{P}$ and $\mathrm{NH}^{\prime} \mathrm{s}$ ist somit eher berechtigt, als eine solche zwischen Kalisalzen und Phosphor ${ }^{1}$ ).

Die anderen stickstoffhaltigen Stoffe zeigen volle $A$ bhängigkeit von der Gesammt-Stickstoffausscheidung. Ich verweise auf das, was bei Stickstoffausscheidung gesagt wurde und auf die unten folgende Tabelle.

Der oben besprochene Fall zeigt kein stetiges Sinken der Temperatur, im Gegentheil sind zwei Recidive zu beobachten; aber gerade dieses war uns willkommen, da wir nicht das Verhalten im Fieber, sondern in Fieberänderungen studiren wollten.

1) So dentet dieses $\mathrm{NH}_{3}$ Verbalten auf die von uns oft hervorgebobene Säureausscheidung, welehe doch meistens als Phosphorsäure auftritt. 


$\begin{array}{ccccccccccc}\text { Temp. } & \text { Cl } & \frac{\mathrm{P}}{\mathrm{N}} & \frac{\mathrm{Ca}}{\mathrm{N}} & \frac{\mathrm{C}}{\mathrm{N}} & \frac{\mathrm{K}}{\mathrm{N}} & \frac{\mathrm{Na}}{\mathrm{N}} & \frac{\text { Harnst. }}{\mathrm{N}} & \frac{\text { Harns. }}{\mathrm{N}} & \frac{\text { Allox. }}{\mathrm{N}} & \frac{\mathrm{NHs}}{\mathrm{N}} \\ 39.1 & 0.107 & 0.065 & 0.0076 & 0.043 & 0.0402 & 0.800 & 0.0099 & 0.0128 & 0.0401 \\ 38.5 & 0.241 & 0.070 & 0.0125 & 0.045 & 0.0845 & 0.862 & 0.0122 & 0.0164 & 0.0542 \\ 39.4 & 0.266 & 0.052 & 0.0046 & 0.051 & 0.0911 & 0.975 & 0.0128 & 0.0182 & 0.0533 \\ 38.6 & 0.108 & 0.077 & 0.0090 & 0.057 & 0.0337 & 0.882 & 0.0119 & 0.0161 & 0.0473 \\ 38.5 & 0.148 & 0.093 & 0.0141 & 0.044 & 0.0385 & 0.838 & 0.0146 & 0.0191 & 0.0553 \\ 39.0 & 0.152 & 0.071 & 0.0089 & 0.056 & 0.0229 & 0.855 & 0.0131 & 0.0160 & 0.0760 \\ 37.5 & 0.098 & 0.063 & 0.0101 & 0.0167 & 0.0176 & 0.668 & 0.0111 & 0.0140 & 0.0700 \\ 38.0 & 0.190 & 0.060 & 0.0075 & 0.0400 & 0.0343 & 0.800 & 0.0159 & 0.0199 & 0.0677 \\ 38.1 & 0.211 & 0.065 & 0.0093 & 0.046 & 0.0526 & 0.864 & 0.0167 & 0.0217 & 0.0703\end{array}$

Tabelle III ist so construirt worden, dass man das Verhältniss von der täglichen Ausscheidung der Harnbestandtheile und Stickstoffausscheidung bildete. Auf diese Weise dargestellt zeigen die Resultate noch deutlicher die Schwankungen, welche bei Erhöhung und Senkung der Temperatur auftreten. So sind die Chloride stets bei Erhöhung des Fiebers vermehrt, bei Sinken verm in dert. Die Phosphate verbalten sich gerade u m g ek ehrt:bei Sinken erhöht, bei Erhöhung vermindert. Das Calcium zeigt sein antagonistisches Verhalten gegenüber dem Temperaturverlanfe auf's deutlichste. Kalium verhält sich auch im Lichte dieser Zahlen unregelmässig und zeigt keine Uebereinstimmung mit Phosphor. Das Ammoniak ist eher mit Chlor in Parallele zu stellen, wenn auch nicht so streng wie das Natrium. Die stickstoffhaltigen Stoffe gehen mehr oder weniger der Temperatur parallel.

Fall III. M. G., 20 Jahre alt. Fibrinöse Pneumonie.

Anamuese. Der Vater des Patienten soll an Tuberculose gestorben sein, die Geschwister seien gesund. Pat. selbst machte bis jetzt keine Krankheit durch. Vor 3 Tagen erkrankte er unter Stechen in der Seite und Schüttelfrosterscheinung.

Status praesens. An den Organen ist nichts Abnormes zu entdecken, ausser einer ausgedehnten Dämpfung in der linken Lungengegend. Die Auscultation bestätigt die Diagnose einer Lungenentzündung. Das Sputum ist rostfarbig und sparsam. Appetit sehr herabgesetzt.

Die Nahrung bestand aus Mileh und Boullion mit Ei. Die Menge wurde genau gemessen.

Die Untersuchung begann gleich nacb der Aufnahme ins Spital. Das Fieber war hoch, 38.8 bis 39.7. Während der Fieberzeit war der Harn gesammelt und auf $\mathrm{N}, \mathrm{Cl}, \mathrm{P}, \mathrm{Ca}$ quantitativ untersucht. Die Wärterin war instruirt, dass sie am Tage der Crise allen Harn vor dem Eintritt des Schweisses besonders auffange; die zweite Portion Harn stammte somit am Tag der Crise von der fieberfreien Periode. An den folgenden Tagen wurde 
die genaue Menge Harn gesammelt und untersucht. Nach dem Eintritt der Crise ereignete es sich, dass die Temperatur nochmals in die Höbe ging, was für unsere Untersuchungen erwünscht war. Dieser Tag wird in der Tabelle ohne weiteres erkenntlich sein. Nach dieser Fieberbewegung fiel dann die Temperatur und blieb bis zum Ende des Versuches normal. Der Harn warde während der Crise und vier Tage nachher auf sämmtliche Bestandtheile quantitativ peprüft; dann noch zwei Tage auf $\mathrm{N}, \mathrm{Cl}, \mathrm{P}, \mathrm{Ca}$.

Im Stuhl wurden Stickstoff, Chlor, Phosphor, Calcium, Kalium und Natrium bestimmt und auf die Gesammtmenge berechnet, dann auf die einzelnen Tage vertheilt.

Die Temperatur verlief, wie folgt:

\begin{tabular}{|c|c|c|c|c|c|c|c|c|c|}
\hline 28. Juli & 38.8 & ois 39.7 & & & Pulszabl & 100 & bis & & \\
\hline 29. Juli & 38.5 & -39.3 & bis & 39.0 & - & 112 & -100 & bis & 104 \\
\hline 30. Juli & 38.1 & -39.5 & - & 39.7 & - & 96 & -100 & - & 99 \\
\hline \multirow[t]{2}{*}{ 31. Juli } & 37.8 & -39.7 & - & 38.8 & - & 96 & 108 & - & 96 \\
\hline & 37.1 & -36.7 & & & - & 92 & 96 & & \\
\hline 1. August & 37.5 & -39.3 & - & 38.0 & - & 96 & 104 & - & 108 \\
\hline 2. August & 37.7 & - 36.2 & & & - & 86 & 84 & & \\
\hline 3. August & 36 & & & & - & 72 & & & \\
\hline 4. August & 36 & & & & - & 70 & & & \\
\hline 5. August & 36 & & & & - & 70 & & & \\
\hline
\end{tabular}

Tabelle IV.

\begin{tabular}{|c|c|c|c|c|c|c|c|c|}
\hline Dat. & $\begin{array}{l}\text { Temp. } \\
\text { und Puls }\end{array}$ & Procent & $\begin{array}{l}\text { Harn- } \\
\text { menge }\end{array}$ & $\begin{array}{l}\text { Spec. } \\
\text { Gew. }\end{array}$ & $\begin{array}{r}\text { Pro Ta } \\
\text { Harn in } g\end{array}$ & $\begin{array}{l}\text { ag } \\
\text { |Koth }\end{array}$ & Nahrung & Bilanz. \\
\hline 8. 7. & $\begin{array}{r}38.8 \\
39.7 \\
100 \\
96\end{array}$ & $\begin{array}{cl}\text { N } & 2.275 \\
\text { Cl } & 0.0607 \\
\text { P } & 0.0500\end{array}$ & & & & & & \\
\hline 29.7. & $\begin{array}{ll}38.5 & 112 \\
39.3 & 100 \\
39.0 & 104\end{array}$ & $\begin{aligned} \text { N } & 2.121 \\
\text { Cl } & 0.0556 \\
\text { P } & 0.0500 \\
\text { Ca } & 0.0109\end{aligned}$ & $\begin{array}{l}\overline{8} \\
8 \\
8 \\
8 \\
8\end{array}$ & 宫 & $\begin{array}{r}21.210 \\
0.556 \\
0.500 \\
0.109\end{array}$ & 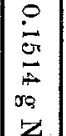 & $\begin{array}{l}2.028 \\
0.533 \\
0.403 \\
0.544\end{array}$ & $\begin{array}{r}-19.333 \\
-\quad 0.025 \\
+\quad 0.181 \\
+\quad 0.223\end{array}$ \\
\hline 30.7 & $\begin{array}{rr}38.1 & 96 \\
39.5 & 100 \\
39.7 & 104\end{array}$ & $\begin{array}{rr}\mathrm{N} & 2.1665 \\
\mathrm{Cl} & 0.0182 \\
\mathrm{P} & 0.0810 \\
\mathrm{Ca} & 0.0016\end{array}$ & $\begin{array}{l}\ddot{8} \\
8 \\
0 \\
0 \\
0\end{array}$ & : & $\begin{array}{r}21.665 \\
0.082 \\
0.810 \\
0.016\end{array}$ & $\begin{array}{l}0 \\
0 \\
0 \\
0 \\
00\end{array}$ & $\begin{array}{l}4.056 \\
1.066 \\
0.806 \\
1.088\end{array}$ & $\begin{array}{r}17.760 \\
+\quad 0.828 \\
-\quad 0.088 \\
+\quad 0.860\end{array}$ \\
\hline 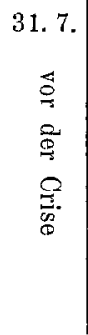 & $\begin{array}{l}37.8 \quad 96 \\
39.7 \quad 108 \\
38.8 \quad 96 \\
6 \text { Uhr } \\
\text { Morgens } \\
\text { bis } 12 \text { Uhr } \\
\text { Nachts }\end{array}$ & $\begin{aligned} \text { N } & 2.170 \\
\text { Cl } & 0.0123 \\
\text { P } & 0.0560 \\
\text { Ca } & 0.0018 \\
\text { K } & 0.1506 \\
\text { Na } & 0.0652 \\
\text { Earnst. } & 1.960 \\
\text { Harns. } & 0.0249 \\
\text { Alloxb. } & 0.0371 \\
\text { Xanth. } & 0.0122 \\
\text { NHs } & 0.1057\end{aligned}$ & 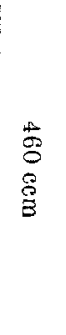 & 宫 & $\begin{array}{l}9.982 \\
0.0566 \\
0.2576 \\
0.0083 \\
0.6927 \\
0.3001 \\
9.016 \\
0.11454 \\
0.17066 \\
0.05612 \\
0.4862\end{array}$ & $\begin{array}{l}0 \\
0 \\
0 \\
0 \\
0 \\
0 \\
0 \\
0 \\
0 \\
0 \\
0 \\
0 \\
0 \\
0 \\
1 \\
19 \\
99 \\
0 \\
0\end{array}$ & $4.056 \mathrm{~N}$ & -18.885 \\
\hline
\end{tabular}


Tabelle IV.

\begin{tabular}{|c|c|c|c|c|c|c|c|c|}
\hline Dat. & $\begin{array}{c}\text { Temp. } \\
\text { und Puls }\end{array}$ & Procent & $\begin{array}{l}\text { Harn- } \\
\text { meng. }\end{array}$ & $\begin{array}{l}\text { Spec. } \\
\text { Gew. }\end{array}$ & $\begin{array}{r}\text { Pro } \mathrm{Ta} \\
\text { Harn in } \mathrm{g}\end{array}$ & & Nahrung & Bilanz. \\
\hline 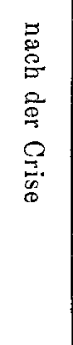 & $\begin{array}{cc}37.1 & 92 \\
36.7 & 96 \\
& \\
12 & \text { Uhr } \\
\text { Nachts } \\
\text { bis } 6 \text { Uhr } \\
\text { Morgens }\end{array}$ & $\begin{array}{rl}\mathrm{N} & 2.247 \\
\mathrm{Cl} & 0.0124 \\
\mathrm{P} & 0.1250 \\
\mathrm{Ca} & 0.0017 \\
\mathrm{~K} & 0.1930 \\
\mathrm{Na} & 0.0178 \\
\text { Harnst. } & 2.030 \\
\text { Harns. } & 0.0182 \\
\text { Alloxb. } & 0.0413 \\
\text { Xantb. } & 0.0231 \\
\mathrm{NHs} & 0.1260\end{array}$ & $\begin{array}{l}0 \\
0 \\
0 \\
8 \\
\beta \\
\beta\end{array}$ & $\dot{8}$ & $\begin{array}{c}12.8079 \\
0.0689 \\
0.7125 \\
0.0097 \\
1.1004 \\
0.1014 \\
11.571 \\
0.1037 \\
0.2314 \\
0.1277 \\
0.7182\end{array}$ & 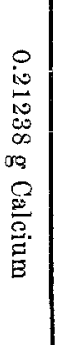 & $\begin{array}{l}1.066 \mathrm{Cl} \\
0.806 \mathrm{P} \\
0.088 \mathrm{Ca} \\
0.920 \mathrm{~K} \\
0.657 \mathrm{Na}\end{array}$ & $\begin{array}{ll}+ & 0.949 \\
+ & 0.248 \\
+ & 0.858 \\
+ & 0.872 \\
+ & 0.256\end{array}$ \\
\hline 1. 8. & $\begin{array}{rr}37.5 & 96 \\
39.3 & 104 \\
38.0 & 108\end{array}$ & $\begin{array}{rr}\mathrm{N} & 2.3520 \\
\mathrm{Ol} & 0.0061 \\
\mathrm{P} & 0.0500 \\
\mathrm{Ca} & 0.0012 \\
\mathrm{~K} & 0.2820 \\
\mathrm{~N} & 0.0138 \\
\text { Harns. } & 0.0217 \\
\text { Alloxb. } & 0.0434 \\
\text { Xanth. } & 0.0217 \\
\text { NHs } & 0.0721\end{array}$ & 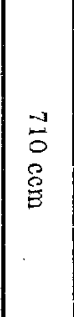 & 5 & $\begin{array}{c}16.7992 \\
0.0433 \\
0.355 \\
0.0085 \\
2.0020 \\
0.0979 \\
0.1541 \\
0.3078 \\
0.1541 \\
0.5419\end{array}$ & & $\begin{array}{l}4.056 \\
1.066 \\
0.806 \\
1.088 \\
0.920 \\
0.657\end{array}$ & $\begin{array}{r}-12.894 \\
+\quad 1.022 \\
+\quad 0.367 \\
+\quad 0.791 \\
+\quad 1.082 \\
+\quad 0.560\end{array}$ \\
\hline 2.7. & $\begin{array}{ll}37.7 & 88 \\
36.2 & 84\end{array}$ & $\begin{array}{rl}\mathrm{N} & 2.352 \\
\mathrm{Cl} & 0.0010 \\
\mathrm{P} & 0.0630 \\
\mathrm{Ca} & 0.0015 \\
\mathrm{~K} & 0.2518 \\
\mathrm{Na} & 0.0376 \\
\text { Harns. } & 0.0157 \\
\text { Alloxb. } & 0.0462 \\
\mathrm{X} \text { anth. } & 0.0305 \\
\mathrm{NHs} & 0.112\end{array}$ & $\begin{array}{l}8 \\
8 \\
8 \\
8\end{array}$ & $\begin{array}{l}5 \\
0 \\
\infty\end{array}$ & $\begin{array}{l}12.760 \\
0.050 \\
0.815 \\
0.0075 \\
1.259 \\
0.188 \\
0.0785 \\
0.231 \\
0.1525 \\
0.560\end{array}$ & 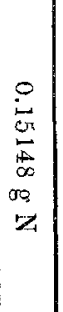 & $\begin{array}{l}4.056 \\
1.066 \\
0.806 \\
1.088 \\
0.920 \\
0.657\end{array}$ & $\begin{array}{r}-\quad 8.955 \\
+\quad 1.059 \\
+\quad 0.093 \\
+\quad 0.769 \\
+\quad 0.339 \\
+\quad 0.469\end{array}$ \\
\hline 3.7. & $36.1 \quad 72$ & $\begin{array}{rl}\mathrm{N} & 2.562 \\
\mathrm{Ol} & 0.0123 \\
\mathrm{P} & 0.1785 \\
\mathrm{Ca} & 0.0016 \\
\mathrm{~K} & 0.2106 \\
\mathrm{Na} & 0.0188 \\
\text { Harnst. } & 2.384 \\
\text { Harns. } & 0.0280 \\
\text { Alloxb. } & 0.0510 \\
\text { Xanth. } & 0.0238 \\
\mathrm{NHs} & 0.1071\end{array}$ & $\begin{array}{l}5 \\
8 \\
8 \\
8 \\
8\end{array}$ & 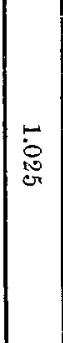 & $\begin{array}{c}25.620 \\
0.1230 \\
0.7850 \\
0.0160 \\
2.1060 \\
0.1880 \\
23.840 \\
0.280 \\
0.510 \\
0.238 \\
1.071\end{array}$ & 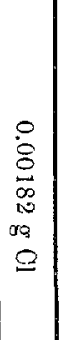 & $\begin{array}{l}4.056 \\
1.066 \\
0.806 \\
1.088 \\
0.920 \\
0.657\end{array}$ & $\begin{array}{rr}-21.715 \\
+\quad 0.941 \\
-\quad 1.063 \\
+\quad 0.861 \\
-\quad 1.186 \\
+\quad 0.469\end{array}$ \\
\hline 4. 7. & $36.2 \quad 70$ & $\begin{array}{rl}\mathrm{N} & 1.8865 \\
\mathrm{Cl} & 0.001 \\
\mathrm{P} & 0.1470 \\
\mathrm{Ca} & 0.0086 \\
\mathrm{~K} & 0.2256 \\
\mathrm{Na} & 0.0468\end{array}$ & $\begin{array}{l}\infty \\
\infty \\
0 \\
8 \\
3 \\
B\end{array}$ & $\dot{0}$ & $\begin{array}{l}15.654 \\
0.0083 \\
1.3201 \\
0.0714 \\
1.867 \\
0.387\end{array}$ & 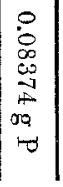 & $\begin{array}{l}2.028 \\
0.533 \\
0.403 \\
0.544 \\
0.460 \\
0.329\end{array}$ & $\begin{array}{r}13.777 \\
+\quad 0.523 \\
+\quad 1.001 \\
+\quad 0.261 \\
+\quad 1.407 \\
+\quad 0.058\end{array}$ \\
\hline
\end{tabular}


Tabelle IV.

\begin{tabular}{|c|c|c|c|c|c|c|c|c|c|}
\hline Dat. & \multicolumn{2}{|c|}{$\begin{array}{l}\text { Temp. } \\
\text { und Puls }\end{array}$} & Procent & \multirow{2}{*}{\begin{tabular}{|c|}
$\begin{array}{c}\text { Harn- } \\
\text { meng. }\end{array}$ \\
\\
\\
$\infty$ \\
0 \\
0 \\
8 \\
8 \\
$\equiv$
\end{tabular}} & \multirow{2}{*}{$\frac{\text { Spec. }}{\text { Gew. }}$} & \multicolumn{2}{|c|}{$\begin{array}{c}\text { Pro Tag } \\
\text { Harn in } \mathrm{g} \mid \text { Koth }\end{array}$} & Nabrung & Bilanz. \\
\hline 4.7. & 36.2 & 70 & $\begin{aligned} \text { Harnst. } & 1.596 \\
\text { Harns. } & 0.01925 \\
\text { Alloxb. } & 0.04450 \\
\text { Xanth. } & 0.02530 \\
\text { NHs } & 0.0700\end{aligned}$ & & & $\begin{array}{l}13.248 \\
0.1577 \\
0.3652 \\
0.2075 \\
0.581\end{array}$ & 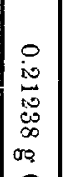 & & \\
\hline 5.7. & 36.1 & 70 & $\begin{array}{cl}\mathrm{N} & 1.995 \\
\mathrm{Cl} & 0.0364 \\
\mathrm{P} & 0.1885 \\
\mathrm{Ca} & 0.0220\end{array}$ & $\begin{array}{l}\infty \\
\dot{1} \\
0 \\
0 \\
0 \\
B\end{array}$ & 若 & $\begin{array}{l}16.517 \\
0.155 \\
0.560 \\
0.1830\end{array}$ & 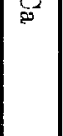 & $\begin{array}{l}2.028 \\
0.533 \\
0.403 \\
0.544\end{array}$ & $\begin{array}{r}-14.644 \\
+\quad 0.376 \\
+\quad 1.241 \\
+\quad 0.314\end{array}$ \\
\hline 6.7. & 36.5 & 70 & $\begin{array}{rl}\mathrm{N} & 1.855 \\
\mathrm{Cl} & 0.6068 \\
\mathrm{P} & 0.121 \\
\mathrm{Ca} & 0.00436\end{array}$ & $\begin{array}{l}\infty \\
\infty \\
0 \\
8 \\
8 \\
B\end{array}$ & $\dot{8}$ & $\begin{array}{r}15.582 \\
5.098 \\
1.016 \\
0.361\end{array}$ & & & \\
\hline 7.7. & 36.2 & 70 & \begin{tabular}{cc|}
$\mathrm{N}$ & - \\
$\mathrm{Cl}$ & 0.7585 \\
$\mathrm{P}$ & 0.0965 \\
$\mathrm{Ca}$ & -
\end{tabular} & $\begin{array}{l}\infty \\
0 \\
0 \\
8 \\
8 \\
B\end{array}$ & 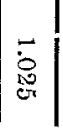 & $\begin{array}{c}-\overline{595} \\
0.839 \\
-\end{array}$ & & & \\
\hline
\end{tabular}

Tabelle V.

Tabelle $\nabla$ giebt die stündliche Ausscheidung wäbrend 8 Tagen wieder.

Dat. Temp. $N \quad \mathrm{Cl} \quad \mathrm{P} \quad \mathrm{Ca} \quad \mathrm{K} \quad \mathrm{Na}$ Harnst. Harns. Allox. NHs 29.7. $38.90 .883 \quad 0.0230 \quad 0.021 \quad 0.0040 \quad-\quad-\quad-\quad-\quad$ $\begin{array}{llllllllllll}30.7 & 39.1 & 0.903 & 0.0080 & 0.034 & 0.0007 & - & - & - & - & -\end{array}$ $\begin{array}{lllllllllllll}31.7 . & 38.8 & 0.554 & 0.0032 & 0.0143 & 0.0005 & 0.0385 & 0.0168 & 0.5009 & 0.0063 & 0.0094 & 0.0270\end{array}$

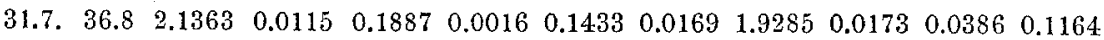
$\begin{array}{llllllllllllllll}\text { 1.8. } & 38.3 & 0.6999 & 0.0018 & 0.0148 & 0.0004 & 0.0867 & 0.0041 & - & 0.0064 & 0.0128 & 0.0213\end{array}$ $\begin{array}{lllllllllllllll}2.8 & 36.9 & 0.500 & 0.0002 & 0.0340 & 0.0003 & 0.0525 & 0.0078 & - & 0.0032 & 0.0096 & 0.0266\end{array}$ $\begin{array}{lllllllllllll}3.8 . & 36.1 & 1.0675 & 0.0051 & 0.0741 & 0.0007 & 0.0875 & 0.0078 & 0.9917 & 0.0127 & 0.0216 & 0.0441\end{array}$ $\begin{array}{lllllllllllll}4.8 . & 36.1 & 0.652 & 0.0003 & 0.0550 & 0.0029 & 0.0775 & 0.0161 & 0.552 & 0.0066 & 0.0153 & 0.0864\end{array}$ 5.8. $36.10 .690 \quad 0.00680 .06500 .0076 \quad-\quad-\quad-\quad-\quad-$ 6.8. $36.10 .649 \quad 0.21090 .04310 .0150 \quad--\quad-\quad-\quad-\quad-\quad-$

Tabelle VI.

Tabelle VI zeigt das Verhältniss der ausgeschiedenen Menge im Verbältniss zur Stickstoffmenge.

Dat. Temp. $\frac{\mathrm{Cl}}{\mathrm{N}} \quad \frac{\mathrm{P}}{\mathrm{N}} \quad \frac{\mathrm{Ca}}{\mathrm{N}} \quad \frac{\mathrm{K}}{\mathrm{N}} \quad \frac{\mathrm{Na}}{\mathrm{N}} \frac{\text { Harnst. Harns. Allox. }}{\mathrm{N}} \frac{\mathrm{NHs}}{\mathrm{N}}$ 29.7. $38.90 .02600 .024 \quad 0.0040 \quad-\quad-\quad-$

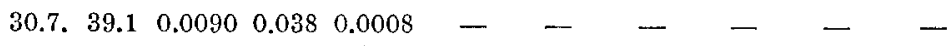

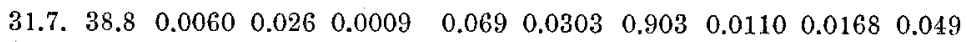
$\begin{array}{lllllllllll}\text { 31.7. } 36.8 & 0.0050 & 0.084 & 0.0007 & 0.067 & 0.0079 & 0.865 & 0.0078 & 0.0178 & 0.054\end{array}$ $\begin{array}{lllllllllllll}\text { 1.8. } 38.3 & 0.0030 & 0.021 & 0.00055 & 0.124 & 0.0058 & - & 0.0091 & 0.0183 & 0.0304\end{array}$ 2.8. $36.9 \quad 0.0003 \quad 0.064 \quad 0.00056 \quad 0.098 \quad 0.004 \quad-\quad 0.0060 \quad 0.0180 \quad 0.042$ 
Dat. Temp. $\frac{\mathrm{Ol}}{\mathrm{N}} \quad \frac{\mathrm{P}}{\mathrm{N}} \quad \frac{\mathrm{Ca}}{\overline{\mathrm{N}}} \quad \frac{\mathrm{K}}{\mathrm{N}} \quad \frac{\mathrm{Na}}{\mathrm{N}} \frac{\text { Harnst. Harns: Allox. }}{\mathrm{N}} \frac{\mathrm{NHs}}{\mathrm{N}}$ $\begin{array}{llllllllll}\text { 3.8. } 36.1 & 0.0049 & 0.069 & 0.0006 & 0.082 & 0.0073 & 0.929 & 0.0118 & 0.0201 & 0.041\end{array}$ $\begin{array}{lllllllllll}\text { 4.8. } & 36.1 & 0.0004 & 0.084 & 0.0044 & 0.118 & 0.024 & 0.800 & 0.0101 & 0.023 & 0.132\end{array}$ $\begin{array}{llllll}5.8 . & 36.1 & 0.0098 & 0.094 & 0.0111\end{array}$

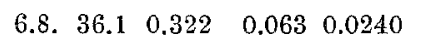

Aus der Taballe IV ersieht man zunächst, dass der Verlust an Stickstoff nach der Crise grösser sein kann als während des Fiebers. Betrachtet man die im Harne ausgeschiedene Menge vor und nach der Crise, so findet man nicht nur den ProcentGehalt, sondern auch die absolute Menge jener Bestandtheile erhöht, deren vermehrte Ausscheidung für das Fieber charakteristisch ist. Noch deutlicher tritt dieses zu Tage, wenn man die stündliche Menge vergleicht. Dabei ergiebt sich (Tabelle V) dass der Stickstoff und das Kalium viermal reichlicher, das Chlor and Calcium dreimal, das Ammoniak fünfmal und das Phosphor achtmal reichlicher ausgeschieden wird, wie vor der Crise. Merkwürdig genug ist die Ausscheidung von $\mathrm{Na}$, so gut wie unverändert, während die stickstoffhaltigen Bestandtheile sämmtlich den anderen folgen, das heisst, vermehrt sind. Es sei betont, dass die Xanthinbasen viel reichlicher ausgeschieden werden, als die Harnsäure, diese zweimal so reichlich, jene viermal. Die ausgeschiedene Menge übertrifft bei Weitem diejenige, welche an den Fiebertagen notirt wurde, auch der Verlust an Stickstoff und Phosphor, aus der Bilanz berechnet, giebt die höchste Zahl für den Tag der Crise. - Berechnet man, wie viel relativ zu $\mathrm{N}$ ausgeschieden wurde, (Tabelle VI), so ist auch hier der erste Platz dem $P$ anzuweisen; $\mathrm{ja}$, die anderen Harnbestandtheile verhalten sich zuweilen anders. So ist das Chlor, Kalium, Natron, Calcium u. s. w., trotzdem dass die stündliche Ausscheidung bedeutend zunahm, doch im Verhältniss zu Stickstoff vermindert, und zwar sind, wie aus der Tabelle VI erhellt Natrium, Chlor, Calcium, Kalium, Harnstoffe, Harnsäure relativ zum Stickstoff vermindert, (das Natrium am bedeutendsten) dagegen Phosphor, Xanthinbasen und Ammoniak vermehrt.

Dieses zur Charakteristik der Crise. Was nun die postfebrile Periode angeht, so ergiebt sich bei jeder Erhöhung der Temperatur ein Sinken der Stickstoff- und Phosphorausscheidung, dagegen ein Steigen der Kalium- und Harnsäureausscheidung. 
- Chlor, Calcium und Natrium folgen dem Stickstoff, sinken somit, wenn die Temperatur in die Höhe geht.

Sinkt die Temperatur, so findet das Umgekehrte statt. Die Stickstoffausscheidung ist vermehrt, ebenso die Phosphor- und Ammoniakausscheidung. In diese Periode fallen auch die grössten Verluste an Stickstoff und Phosphor, welche die Bilanz ergiebt. Das Chlor verhält sich unregelmässig, oft steigt es an den fieberfreien Tagen, dann fällt es aber bis zu solchen Zahlen, welche auf der Fieberhöhe nie beobachtet wurden. Es ergiebt sich aus der Bilanz; dass die Retention im Organismus nach dem Fieberabfall die grösste ist und dass sie allmählich abnimmt; ebenso verbält sich die Natrium- und Calciumbilanz. - Phosphor wird dagegen nach dem Fieberabfall der Körper mehr verlustig, und dieser P-Verlust steigt nach dem Fieber immer noch an, bis er nach drei bis vier Tagen zu sinken anfängt. Die Kalisalze werden vom Körper verloren, und zwar am geringsten gleich nach dem Fieber, dann bedeutender.

Ungefähr das Gleiche ergiebt sich aus der Analyse der Tabelle der stündlichen Ausscheidung, nur dass hier die Abhängigkeit von der Temperaturbewegung deutlicher zu Tage tritt.

Noch deutlicher zeigt es sich bei der Tabelle VI, wo die stündliche Ausscheidung im Verhältniss zum Stickstoff berechnet ist. Hier zeigt das Verhalten des Phosphors die grösste Regelmässigkeit, Chlor ist undeatlich, ebenso die Kali- und Natronsalze.

Es ist gar nicht zu bestreiten, dass sich das Chlor, Calcium und Kalium ganz anders im Typhus, als hier verhält, und darin mag es liegen, dass die Resultate der Fieberuntersuchungen so verschieden lauten.

Fall IV. H. K., 19 Jahre alt. Febris intermittens quotidiana.

Anamnese. Der Vater des Patienten starb an der Cholera, die Mutter ist kränklich. Drei Geschwister starben im Jugendalter. Patient stammt aus Hamburg, hielt sich in Italien auf, woselbst er zuerst an Fieber und Kopfweh erkrankte. Er verliess sodann dieses Land und begab sich in die Schweiz, während der Fussreise litt er an Schüttelfrost, welchen er früber in Italien bei den Fieberanfällen nicht hatte.

St. praesens. An den Organen ist ansser einer leichten Milzvergrösserung nichts zu entdecken. Haemoglobingehalt 60 pCt, Blutkzhl. 4.120.000.

Die Nahrung bestand aus Milch und Bouillon und betrug pro Tag
$10.340 \mathrm{~g}$ Stickstoff
$6.246 \mathrm{~g}$ Chlor
1.260 - Phosphor
1.740 - Calcium
2.748 - Kalium
3.562 - Natrium 
Der Harn wurde in fünf Portionen gefangen. Die erste begriff die Zeit vor dem Fieber $10 \mathrm{~h}^{\prime}$ Vormittags bis $2 \frac{1}{2} \mathrm{~h}^{\prime}$ Nachmittags. Temp. 36.4. Pulszahl 92. Harnmenge $125 \mathrm{ccm}$.

Die zweite umfasste die Zeit des Schüttelfrostes $2 \frac{1}{2} \mathrm{~b}^{\prime}$ Nachmittags bis 4 h' Nachmittags. Temp. 41.0. Pulszahl 106. Harnmenge 235 cem.

Die dritte umfasste die Zeit der Fieberböhe $4 \mathrm{~h}^{2}$ Nachmittags bis $7 \mathrm{~h}^{\prime} \mathrm{Nach}$ mittags. Temp. 40.2. Pulszahl 96. Harnmenge $295 \mathrm{ccm}$.

Die vierte begriff die Zeit nach dem Fieberanfall $7 \mathrm{~h}^{\prime}$ Nachmittags bis $6 h^{\prime}$ Morgens. Temp. 39.938 .6 37.5. Pulszahl 108 104 96. Harnmenge $325 \mathrm{ccm}$.

Die fünfte and letzte Portion begriff die Zeit der normalen Temperatur 6 b' Morgens bis 10 h' Vormittags. Temp. 36.3. Pulszahl 80. Harnmenge 185 .

Der Patient schied $1165 \mathrm{ccm}$ Harn aus und darin:

$\begin{array}{rrr}\mathrm{N} & 15.792 & \text { Bilanz } \\ \mathrm{Cl} & 4.934 & \mathrm{~N}-5.452 \\ \mathrm{P} & 0.917 & \mathrm{Cl}+1.312 \\ \mathrm{Ca} & 0.263 & \mathrm{P}+0.343 \\ \mathrm{~K} & 2.665 & \mathrm{Ca}+1.477 \\ \mathrm{Na} & 2.739 & \mathrm{~K}+0.083 \\ & \mathrm{Na}+0.823\end{array}$

Die Nahrung enthielt die oben angegebene Menge, somit zeigte die Bilanz wie folgende Tabelle ergiebt, für Stickstoff einen Verlust. Alle anderen Bestandtheile waren retinirt, was insofern einer Correctur bedarf, als der Koth nicht berücksichtigt wurde. Dieses betrifft hauptsächlich $\mathrm{P}, \mathrm{Ca}, \mathrm{K}$. Hält man sich an die bekannten Zablen, so kann wan hier einen geringen Verlust an $P$ und Kalium vermuthen, das Calcium, Natrium and Chlor bleiben wohl obne Aenderung.

In der Nahrung gestaltet sich das Verbältniss zu Stickstoff:
$\mathrm{N}: \mathrm{CI}: \mathrm{P}: \mathrm{Ca}: \mathrm{K}: \mathrm{Na}$
$\begin{array}{llllll}1: 0.62 & 0.12 & 0.17 & 0.27 & 0.35 & \text { in der Nahrung. }\end{array}$
$1: 0.3120 .058 \quad 0.016 \quad 0.1610 .165$ in der Ansscheidung.

Somit sind gegenüber dem Stickstoff alle Bestandthejle vermindert ausgeschieden.

Die stündliche Ausscheidnng wird in der Tabelle VII Platz finden.

Tabelle VII. Malariafieber.

\begin{tabular}{|c|c|c|c|c|c|c|c|}
\hline Zeit & $\begin{array}{l}\text { Temp. } \\
\text { u. Puls }\end{array}$ & Proc. & $\begin{array}{l}\text { Harn- } \\
\text { meng. }\end{array}$ & $\begin{array}{l}\text { Spec. } \\
\text { Gew. }\end{array}$ & $\begin{array}{l}\text { Gesammt- } \\
\text { ausscheidung }\end{array}$ & $\begin{array}{l}\text { Pr. Std. } \\
\text { Gramm }\end{array}$ & Zeit. \\
\hline I. & $\begin{array}{c}36.4 \\
92\end{array}$ & $\begin{array}{l}1.6205 \\
0.2912 \\
0.0891 \\
0.0484 \\
0.1522 \\
0.1377\end{array}$ & 宓 & $\begin{array}{l}10 \\
8 \\
8 \\
8 \\
8\end{array}$ & $\begin{array}{rr}\mathrm{N} & 2.025 \\
\mathrm{Cl} & 0.364 \\
\mathrm{P} & 0.111 \\
\mathrm{Ca} & 0.061 \\
\mathrm{~K} & 0.190 \\
\mathrm{Na} & 0.172\end{array}$ & $\begin{array}{l}0.455 \\
0.081 \\
0.025 \\
0.014 \\
0.042 \\
0.038\end{array}$ & $\begin{array}{l}\text { 10 U. Vorm.bis } \\
2 \frac{1}{2} \text { U. Nachm. } \\
4.5 \text { Stunden. } \\
\text { Harnmenge } \\
\text { pro Stunde } \\
27.7 \mathrm{ccm} .\end{array}$ \\
\hline
\end{tabular}




\begin{tabular}{|c|c|c|c|c|c|c|c|}
\hline$Z_{\text {eit }}$ & $\begin{array}{l}\text { Temp. } \\
\text { u. Puls }\end{array}$ & Proc. & $\begin{array}{l}\text { Harn- } \\
\text { meng. }\end{array}$ & $\begin{array}{l}\text { Spec. } \\
\text { Gew. }\end{array}$ & $\begin{array}{l}\text { Gesammat- } \\
\text { ausscheidung }\end{array}$ & $\begin{array}{l}\text { pr. Std. } \\
\text { Gramm }\end{array}$ & Zeit. \\
\hline II. & $\begin{array}{r}41.0 \\
106\end{array}$ & $\begin{array}{l}0.7875 \\
0.6371 \\
0.0162 \\
0.0171 \\
0.2390 \\
0.3676 \\
0.0259\end{array}$ & $\underset{\infty}{\infty}$ & $\begin{array}{l}\mathrm{N} \\
\mathrm{S} \\
\mathrm{G} \\
\mathrm{E} \\
\mathrm{B}\end{array}$ & $\begin{array}{rr}\mathrm{N} & 1.8485 \\
\mathrm{Cl} & 1.4969 \\
\mathrm{P} & 0.0376 \\
\mathrm{Ca} & 0.0399 \\
\mathrm{~K} & 0.5497 \\
\mathrm{Na} & 0.8454 \\
\mathrm{NHs} & 0.0596\end{array}$ & $\begin{array}{l}1.2325 \\
0.9969 \\
0.0251 \\
0.0266 \\
0.3665 \\
0.5636 \\
0.0397\end{array}$ & $\begin{array}{l}2 \frac{1}{2} \text { U. Nachm. } \\
\text { b. } 4 \text { U. Nachm. } \\
\text { 1.5 Stunden. } \\
\text { Earnmenge } \\
\text { pro Stunde } \\
94 \mathrm{ccm} .\end{array}$ \\
\hline III. & $\begin{array}{c}40.0 \\
92\end{array}$ & $\begin{array}{l}0.183 \\
0.4854 \\
0.0043 \\
0.0252 \\
0.1884 \\
0.2584 \\
0.0105 \\
0.0109 \\
0.0004 \\
0.0266\end{array}$ & $\underset{0}{\stackrel{0}{0}}$ & $\begin{array}{l}N \\
0 \\
0 \\
0 \\
O \\
B\end{array}$ & $\begin{array}{rl}\mathrm{N} & 3.481 \\
\mathrm{Cl} & 1.416 \\
\mathrm{P} & 0.0127 \\
\mathrm{Ca} & 0.0737 \\
\mathrm{~K} & 0.5546 \\
\mathrm{Na} & 0.7611 \\
\text { Harns. } & 0.0309 \\
\text { Alloxb. } & 0.0321 \\
\text { Xanth. } & 0.0018 \\
\text { NHs } & 0.0784\end{array}$ & $\begin{array}{l}1.160 \\
0.472 \\
0.0042 \\
0.0246 \\
0.1849 \\
0.2534 \\
0.0103 \\
0.0107 \\
0.0004 \\
0.0961\end{array}$ & $\begin{array}{l}4 \text { U. Nachm. } \\
\text { b. } 7 \text { U. Abends } \\
3 \text { Stunden. } \\
\text { Harnmenge } \\
\text { pro Stunde } \\
95 \text { cem. }\end{array}$ \\
\hline IV. & $\begin{array}{c}39.9 \\
38.6 \\
37.5 \\
108 \\
104 \\
97\end{array}$ & $\begin{array}{l}1.645 \\
0.3034 \\
0.1458 \\
0.0142 \\
0.2916 \\
0.1798 \\
1.120 \\
0.0245 \\
0.0249 \\
0.0004 \\
0.0560\end{array}$ & $\begin{array}{l}\bar{O} \\
\mathbb{N}\end{array}$ & 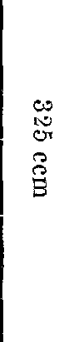 & $\begin{array}{rl}\mathrm{N} & 5.3302 \\
\mathrm{CI} & 0.9847 \\
\mathrm{P} & 0.4747 \\
\mathrm{Ca} & 0.0455 \\
\mathrm{~K} & 0.9457 \\
\mathrm{Na} & 0.5854 \\
\text { Harnst. } & 3.640 \\
\text { Harns. } & 0.0796 \\
\text { Alloxb. } & 0.0809 \\
\text { Xanth. } & 0.0013 \\
\mathrm{NHs} & 0.1810\end{array}$ & $\begin{array}{l}0.4845 \\
0.0895 \\
0.0432 \\
0.0041 \\
0.0859 \\
0.0532 \\
0.3310 \\
0.0072 \\
0.0073 \\
0.0001 \\
0.0165\end{array}$ & $\begin{array}{c}7 \text { U. Nachm. } \\
\text { bis } 6 \text { U. Mlorg. } \\
11 \text { Stunden. } \\
\text { Harnmenge } \\
29.5 \text { ecm. }\end{array}$ \\
\hline V. & $\begin{array}{c}36.3 \\
80\end{array}$ & $\begin{array}{l}1.687 \\
0.3641 \\
0.0512 \\
0.9234 \\
0.2208 \\
0.2032 \\
0.624\end{array}$ & ${ }_{0}$ & $\begin{array}{l}\text { D. } \\
0 \\
0 \\
8 \\
0 \\
B\end{array}$ & $\begin{array}{rr}\mathrm{N} & 3.1080 \\
\mathrm{Cl} & 0.6734 \\
\mathrm{P} & 0.2817 \\
\mathrm{Ca} & 0.0433 \\
\mathrm{~K} & 0.4255 \\
\mathrm{Na} & 0.3759 \\
\text { Harnst. } & 2.997\end{array}$ & $\begin{array}{l}0.7770 \\
0.1683 \\
0.0704 \\
0.0108 \\
0.1064 \\
0.0940 \\
0.7490\end{array}$ & $\begin{array}{c}6 \text { U. Morg. bis } \\
10 \text { U. A bends } \\
4 \text { Stunden } \\
\text { Harnmenge } \\
46.2 \text { cem. }\end{array}$ \\
\hline
\end{tabular}

\section{Tabelle VIII.}

\begin{tabular}{|c|c|c|c|c|c|c|c|c|c|}
\hline Temn. & $\mathrm{Cl}$ & P & $\underline{\mathrm{Ca}}$ & K & $\mathrm{Na}$ & Harnst. & NEs & Barns. & Allox. \\
\hline & $\overline{\mathrm{N}}$ & & $\overline{\mathrm{N}}$ & & $\overline{\mathrm{N}}$ & $\mathrm{N}$ & $\overline{\mathrm{N}}$ & $\overline{\mathrm{N}}$ & $\mathrm{N}$ \\
\hline 36.4 & 0.178 & 0.056 & 0.0307 & 0.092 & 0.083 & - & - & - & - \\
\hline 41.0 & 0.809 & 0,020 & 0.0215 & 0.297 & 0.457 & 0.996 & 0.032 & - & - \\
\hline 40.0 & 0.407 & 0.003 & 0.0212 & 0.159 & 0.218 & - & 0.0224 & 0.009 & 0.009 \\
\hline 38.6 & 0.184 & 0.089 & 0.0084 & 0.117 & 0.109 & 0.660 & 0.034 & 0.014 & 0.005 \\
\hline 36.3 & 0.215 & 0.091 & 0.0139 & 0.137 & 0.121 & - & - & - & - \\
\hline
\end{tabular}

Die Resultate dieser Untersuchung bieten so wenig Neues, dass man die Besprechung kaum halten kann. Was Limbeck, Terray, Hitzig und andere gefunden, wurde bestätigt. 
Die Stickstoffausscheidung stieg und fiel mit der Temperatur aber nur absolut genommen. Relativ zur Wasserausscheidung war sie während des Fiebers vermindert, wie aus dem Procentgehalt an Stickstoff zu ersehen ist.

Die Chlorausscheidung stieg während des Fiebers ganz bedeutend relativ und absolut, verminderte sich gleich nach dem Abfall des Fiebers um dann während der Apyrexie zu steigen.

Die Phosphorausscheidung dagegen fiel während des Fiebers und erreichte in der postfebrilen Periode ihr Maximum. In der postfebrilen Periode war die P-Ausscheidung normal.

Die Ausscheidung von Kalisalzen war absolut der des Chlors völlig analog, wenngleich relativ in der Wasserausscheidung im Fieberanfang ein Sinken zu beobachten war, welches während des Fiebers anhielt.

Die Kalisalze nehmen wie immer eine Sonderstellung ein. Relativ zur Wasserausscheidung erreichen sie ihr Maximum in der postfebrilen Periode. Absolut genommen folgen sie dem Chlor and haben ihr Minimum in der postfebrilen Periode. Die. Natronsalze sind in allen Punkten mit Chlor zu vergleichen.

Die Ausscheidung der stickstoffhaltigen Stoffe konnte wegen Materialmangel nicht überall bestimmt werden, deshalb ist über ihr Verhalten schwer etwas auszusagen. Es scheint, als ob nach dem Fieber die Ausscheidung der Harnsäure und der Xanthinbasen relativ grösser wurde zu der Wasserausscheidung and zum $\mathrm{N}$, wenn auch die absolute Menge pro Stunde in der postfebrilen Periode geringer ist.

Setzt man die stündlich ausgeschiedenen Mengen im Verhältniss zu Stickstoff, so ergiebt sich alles oben Gesagte noch deutlicher.

Die tägliche Ausscheidung im Vergleich zur Nahrungsaufnahme ergiebt das typische Verhalten im Fieber, eine relativ verminderte Chlor-, Calcium- und Natriumausscheidung und vermehrte N-, P- und Kaliausscheidung.

Fall V. B. K., 49 Jahre alt. Phtisis pulmonum Febris hectiea.

Anamnese. Der Vater und die Gesehwister sind gesund, die Mutter soll an Husten gelitten haben. Patientin machte eine Gonitis durch. Sie heirathete und hatte zwei Kinder, dieselben starben im Kindesalter. Das jetzige Leiden begann vor wenigen Monaten und brachte allgemeine Sehwäche mit sicb.

St. praesens. Patientin zeigt in beiden Lungen die Erseheinungen einer zerfallenden Infiltration, wirft viel aus. Die anderen Organe bieten 
nichts Abnormes. Patientin fiebert im hektischen Typus. Der Temperaturunterschied beträgt $2,5^{\circ} \mathrm{C}$.

Patientin bekam während mehrerer Tage die gleiche Diät, bestebend aus Milch und Bouillon. Der Harn wurde an zwei Tagen analysirt, obne dass dabei Unterschiede bemerkt wurden. Die tägliche Ausscheidung wurde in 4 Portionen gesammelt, wovon

die erste von 12 Uhr Mittags bis 4 Uhr Nachmittags als der praefebrilen Periode entsprechend, gesammelt wurde. Die Temperatur betrug 37.0, Pulszabl 100,

die zweite Portion umfasst die Periode des Fiebers von 4 Uhr Nachmittags bis 10 Uhr Nachts. Temperatar 38.0 bis 39.0 ,

die dritte Periode entspricht dem Fieberabfall von 10 Uhr Nachts bis 6 Uhr Morgens. Temperatur 37.0,

die vierte und letzte dauerte von 6 Uhr Morgens bis 12 Uhr Mittags und war die Periode der Apyrexie. Temperatur 36.0, Pulszahl 90.

Auch bier wurde der Koth nicht untersucht, was aber die uns hier beschäftigende Frage nichts angeht. Die Resultate der Zusammenstellung ergaben:

$\begin{array}{lcc}\text { Ausgaben } & \text { Nahrung } & \text { Bilanz } \\ 5.6006 \mathrm{~N} & 10.340 & +4.7394 \mathrm{gr} \mathrm{N} \\ 3.7664 \mathrm{Cl} & 6.246 & +2.4796-\mathrm{Ol} \\ 0.2749 \mathrm{P} & 1.260 & +0.9851-\mathrm{P} \\ 0.0952 \mathrm{Ca} & 1.740 & +1.6448-\mathrm{Ca} \\ 1.0642 \mathrm{~K} & 2.748 & +1.6840-\mathrm{K} \\ 1.1028 \mathrm{Na} & 3.562 & +2.4592-\mathrm{Na}\end{array}$

Das Verhältniss des $\mathrm{N}$ zu $\mathrm{Cl}, \mathrm{P}, \mathrm{Ca}, \mathrm{K},{ }^{\circ} \mathrm{Na}$ in der Nahrung war:

$\begin{array}{clcccc} & \mathrm{N}: \mathrm{Cl}: & \mathrm{P}: & \mathrm{Ca}: & \mathrm{K}: & \mathrm{Na}: \\ & 1: 0.62 & 0.12 & 0.17 & 0.27 & 0.35 \\ \text { in der Ausscheidung } & 1: 0.67 & 0.04 & 0.016 & 0.19 & 0.20\end{array}$

Der Unterschied betrifit $\mathrm{Ca}$ und $\mathrm{P}$, beide sind im Koth reichlich vertreten. Wir dürfen also.annehmen, dass keine spec. Retention hier stattfinde, nur eine allgemeine Apathie, mangelhafte Assimilation vorliege.

Tabelle IX.

\begin{tabular}{|c|c|c|c|c|c|c|c|}
\hline Zeit & $\begin{array}{l}\text { Temp. } \\
\text { u. Puls }\end{array}$ & $\begin{array}{l}\text { Harn- } \\
\text { meng. }\end{array}$ & $\begin{array}{l}\text { Spec. } \\
\text { Gew. }\end{array}$ & Procent & $\begin{array}{l}\text { Gesammt- } \\
\text { ausscheid. }\end{array}$ & pr. Stud. & $\begin{array}{l}\text { Wasser } \\
\text { pro Std. }\end{array}$ \\
\hline $\begin{array}{l}12 \text { U. Mitt. } \\
\text { bis } \\
4 \text { U. Nachm. } \\
4 \text { Stunden. }\end{array}$ & $\begin{array}{l}37^{0} \\
100\end{array}$ & 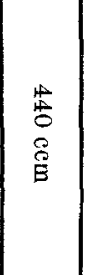 & 묭 & $\begin{array}{rl}\mathrm{N} & 0.217 \\
\mathrm{Cl} & 0.1214 \\
\mathrm{P} & 0.0235 \\
\mathrm{Ca} & 0.0049 \\
\mathrm{~K} & 0.0401 \\
\mathrm{Na} & 0.0515 \\
\text { Harnst. } & 0.196 \\
\text { Alloxb. } & 0.0075 \\
\mathrm{NHs} & 0.0056\end{array}$ & $\begin{array}{l}0.9548 \\
0.5342 \\
0.1034 \\
0.0215 \\
0.1764 \\
0.2860 \\
0.8624 \\
0.0430 \\
0.0246\end{array}$ & $\begin{array}{l}0.2387 \\
0.1335 \\
0.0259 \\
0.0054 \\
0.0441 \\
0.0715 \\
0.2156 \\
0.0108 \\
0.0061\end{array}$ & $\begin{array}{c}\text { T. } \\
110 \mathrm{cem}\end{array}$ \\
\hline
\end{tabular}


Tabelle IX.

\begin{tabular}{|c|c|c|c|c|c|c|c|}
\hline Zeit & $\begin{array}{l}\text { Temp. } \\
\text { a. Puls }\end{array}$ & $\begin{array}{l}\text { Aarn- } \\
\text { meng. }\end{array}$ & $\mid \begin{array}{l}\text { Spec. } \\
\text { Gew. }\end{array}$ & Procent & $\begin{array}{l}\text { Gesammt- } \\
\text { ausscheid. }\end{array}$ & $\begin{array}{l}\text { Pr. Std. } \\
\text { Gramm }\end{array}$ & $\begin{array}{l}\text { Wasser } \\
\text { pro Std. }\end{array}$ \\
\hline $\begin{array}{l}4 \text { U. Nachm. } \\
\text { bis } 10 \mathrm{U} . \\
\text { Abends } \\
6 \text { Stunden. }\end{array}$ & $\left|\begin{array}{c}38-39 \\
106\end{array}\right|$ & $\begin{array}{l}\stackrel{1}{8} \\
8 \\
3 \\
8 \\
8\end{array}$ & $\stackrel{\dot{\rho}}{0}$ & $\begin{array}{rl}\mathrm{N} & 0.378 \\
\mathrm{Cl} & 0.315 \\
\mathrm{P} & 0.0290 \\
\mathrm{Ca} & 0.0068 \\
\mathrm{~K} & 0.0629 \\
\mathrm{Na} & 0.0852 \\
\text { Harnst. } & 0.341 \\
\text { Harns. } & 0.0077 \\
\text { Alloxb. } & 0.0109 \\
\text { Xanth. } & 0.0032 \\
\text { NH's } & 0.0119\end{array}$ & $\begin{array}{l}0.7388 \\
1.4490 \\
0.1335 \\
0.0312 \\
0.2893 \\
0.3919 \\
0.5686 \\
0.0354 \\
0.0502 \\
0.0148 \\
0.0510\end{array}$ & $\begin{array}{l}0.2898 \\
0.2415 \\
0.0223 \\
0.0052 \\
0.0482 \\
0.0653 \\
0.2614 \\
0.0060 \\
0.0084 \\
0.0024 \\
0.0085\end{array}$ & $\begin{array}{l}\text { II. } \\
76.6 \mathrm{ccm}\end{array}$ \\
\hline $\begin{array}{l}10 \mathrm{U} . \\
\text { Abends bis } \\
6 \text { U. Morg. } \\
8 \text { Stunden. }\end{array}$ & $\begin{array}{l}37.1 \\
100\end{array}$ & $\begin{array}{l}\stackrel{A}{\circ} \\
\circ \\
8 \\
B \\
B\end{array}$ & $\stackrel{\infty}{\varnothing}$ & $\begin{array}{rl}\mathrm{N} & 0.392 \\
\mathrm{Cl} & 0.2669 \\
\mathrm{P} & 0.0160 \\
\mathrm{Ca} & 0.0078 \\
\mathrm{~K} & 0.0829 \\
\mathrm{Na} & 0.0590 \\
\text { Harnst. } & 0.322 \\
\text { Harns. } & 0.0081 \\
\text { Alloxb. } & 0.0116 \\
\text { Xantb. } & 0.0035 \\
\text { NHs } & 0.0097\end{array}$ & $\begin{array}{l}1.764 \\
1.201 \\
0.072 \\
0.0351 \\
0.3731 \\
0.2655 \\
1.449 \\
0.0365 \\
0.0522 \\
0.0158 \\
0.0437\end{array}$ & $\begin{array}{l}0.2205 \\
0.1501 \\
0.0090 \\
0.0043 \\
0.0441 \\
0.0332 \\
0.1811 \\
0.0046 \\
0.0063 \\
0.0019 \\
0.0055\end{array}$ & $\begin{array}{c}\text { III. } \\
56 \mathrm{ccm}\end{array}$ \\
\hline $\begin{array}{l}6 \text { U. Morg. } \\
\text { bis } \\
12 \text { U. Mitt. } \\
6 \text { Stunden. }\end{array}$ & 36.0 & $\begin{array}{l}\text { N } \\
\stackrel{心}{0} \\
8 \\
8 \\
B\end{array}$ & $\dot{0}$ & $\begin{array}{rl}\mathrm{N} & 0.476 \\
\mathrm{Cl} & 0.2427 \\
\mathrm{P} & 0.0275 \\
\mathrm{Ca} & 0.0031 \\
\mathrm{~K} & 0.0939 \\
\mathrm{Na} & 0.664 \\
\mathrm{NH} & 0.0084\end{array}$ & $\begin{array}{l}1.1424 \\
0.5822 \\
0.0660 \\
0.0074 \\
0.2254 \\
0.1594 \\
0.0202\end{array} \mid$ & $\begin{array}{l}0.1904 \\
0.0970 \\
0.0110 \\
0.0012 \\
0.0376 \\
0.0266 \\
0.0033\end{array}$ & $\begin{array}{l}\text { IV. } \\
40 \mathrm{ccm}\end{array}$ \\
\hline & & & & weiter Tag. & & & \\
\hline $\begin{array}{l}12 \text { U. Mitt. } \\
\text { bis } \\
4 \text { U. Nachm. } \\
4 \text { Stunden. }\end{array}$ & $\begin{array}{c}370 \\
90\end{array}$ & $\begin{array}{l}10 \\
\infty \\
0 \\
8 \\
8 \\
0\end{array}$ & - & $\begin{aligned} & \text { N } 0.385 \\
& \text { Ol } 0.255 \\
& \text { P } 0.038 \\
& \text { Harns. } 0.0081 \\
& \text { Alloxb. } 0.0115 \\
& \text { Xanth. } 0.0037 \\
& \text { NHs } 0.0105\end{aligned}$ & $\begin{array}{l}1.0780 \\
0.7140 \\
0.1064 \\
0.0225 \\
0.0329 \\
0.0104 \\
0.0294\end{array}$ & $\begin{array}{l}0.269 \\
0.179 \\
0.0266 \\
0.0056 \\
0.0082 \\
0.0026 \\
0.0074\end{array}$ & $\begin{array}{c}\mathrm{I} \\
70 \mathrm{ccm}\end{array}$ \\
\hline $\begin{array}{c}4 \text { U. Nachm. } \\
\text { bis } \\
10 \text { U. Abds. } \\
6 \text { Stuuden. }\end{array}$ & $39^{\circ}$ & $\begin{array}{l}\stackrel{0}{2} \\
\text { O } \\
0 \\
\text { B }\end{array}$ & 一 & $\begin{aligned} \text { N } & 0.518 \\
\text { Cl } & 0.2851 \\
\text { P } & 0.035 \\
\text { Harns. } & 0.0103 \\
\text { Alloxb. } & 0.0109 \\
\text { Xanth. } & 0.0006 \\
\text { NHs } & 0.0133\end{aligned}$ & $\begin{array}{l}1.8648 \\
1.0260 \\
0.1260 \\
0.0371 \\
0.0392 \\
0.0022 \\
0.0479\end{array}$ & $\begin{array}{l}0.3108 \\
0.1710 \\
6.021 \\
0.0062 \\
0.0065 \\
0.0003 \\
0.0080\end{array}$ & $\begin{array}{l}\text { II. } \\
60 \mathrm{ccm}\end{array}$ \\
\hline
\end{tabular}


Tabelle IX.

\begin{tabular}{|c|c|c|c|c|c|c|c|}
\hline Zeit & $\begin{array}{l}\text { Temp. } \\
\text { u. Puls }\end{array}$ & Harn- & $\begin{array}{l}\text { Spec. } \\
\text { Gew. }\end{array}$ & Procent & $\begin{array}{l}\text { Gesammt- } \\
\text { ausscheid. }\end{array}$ & $\begin{array}{l}\text { pr. Std. } \\
\text { Gramm }\end{array}$ & $\begin{array}{l}\text { Wasser } \\
\text { pro Std. }\end{array}$ \\
\hline $\begin{array}{l}10 \text { U. Abds. } \\
\text { bis } \\
6 \text { U. Morg. } \\
8 \text { Stunden. }\end{array}$ & $38^{\circ}$ & $\begin{array}{l}0 \\
0 \\
0 \\
8 \\
8 \\
B\end{array}$ & 一 & $\begin{array}{rr}\mathrm{N} & 0.5145 \\
\mathrm{Cl} & 0.2548 \\
\mathrm{P} & 0.0320 \\
\text { Harns. } & 0.0116 \\
\text { Alloxb. } & 0.0126 \\
\text { Xanth. } & 0.0010 \\
\text { NHs } & 0.0168\end{array}$ & $\begin{array}{l}1.286 \\
0.637 \\
0.080 \\
0.0290 \\
0.0395 \\
0.0025 \\
0.0420\end{array}$ & $\begin{array}{l}0.161 \\
0.079 \\
0.010 \\
0.0036 \\
0.0039 \\
0.0003 \\
0.0052\end{array}$ & $\begin{array}{c}\text { III. } \\
31 \mathrm{ccm}\end{array}$ \\
\hline $\begin{array}{l}6 \text { U. Morg. } \\
\text { bis } \\
12 \text { U. Mitt. } \\
6 \text { Stunden. }\end{array}$ & 36 & $\begin{array}{l}\text { c } \\
0 \\
8 \\
\Xi \\
\Xi\end{array}$ & - & $\begin{array}{rl}\mathrm{N} \\
\mathrm{Cl} & 0.2366 \\
\text { Harns. } & 0.0131 \\
\text { Alloxb. } & 0.0205 \\
\text { Xanth. } & 0.0073\end{array}$ & $\begin{array}{c}-\overline{1183} \\
0.0065 \\
0.0102 \\
0.0036\end{array}$ & $\begin{array}{c}-\overline{-} \\
0.0197 \\
0.0011 \\
0.0017 \\
0.0006\end{array}$ & $\begin{array}{l}\text { IV. } \\
9 \mathrm{ccm}\end{array}$ \\
\hline
\end{tabular}

Tabelle X.

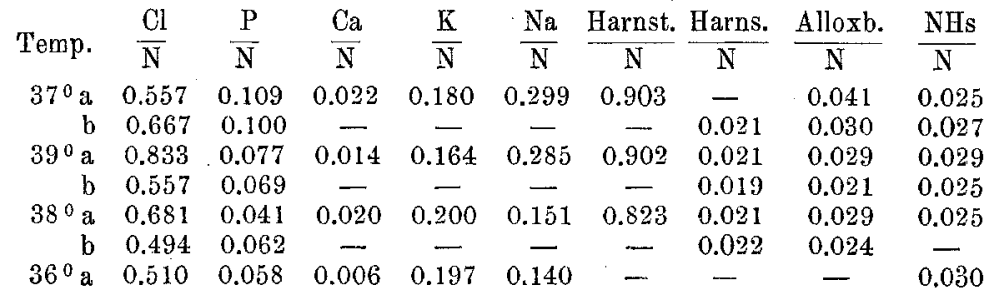

Die Ergebnisse der Beobachtung der Ausscheidung beim hektischen Fieber bestätigen die bei Malaria notirten Resultate. Auch hier ist die Chlorausscheidung bei der Fieberperiode geringer als in der fieberfreien Zeit. Das Minimum liegt in der Apyrexie. Die Phosphor-Ausscheidung fällt mit der zunehmenden Temperatur, erreicht ihr Minimum in der postfebrilen Zeit und steigt dann in der Periode der Apyrexie bis zur praefebrilen Zeit, wo sie ihr Maximum erreicht.

Das Calcium fällt mit Beginn des Fiebers, nimmt dann noch etwas $a b$ und fällt in der Apyrexie auf das Minimum. Das Maximum liegt in der praefebrilen Zeit.

Das Natrium geht hier merkwürdig genug dem Chlor nicht parallel. Es sinkt auf der Fieberhöhe, sinkt dann noch mehr, erreicht das Minimum in der postfebrilen Zeit nicht (übereinstimmend mit Chlor) und das Maximum in der praefebrilen Periode. - Das Kalium steigt mit dem Fieber, fällt dann bis zur Apyrexie stetig, wo es sein Minimum erreicht, während das 
Maximum auf der Fieberhöhe liegt. - Der Harnstoff wird auf der Fieberhöhe am reichlichsten ausgeschieden, nicht so die Alloxurbasen, welche in der praefebrilen Periode am wenigsten zur Ausscheidung kommen, während ihr Maximum in der Apyrexie liegt.

Das Ammoniak wird wie das Chlor während der Fieberhöhe mehr ausgeschieden, sinkt dann, erreicht in der Apyrexie sein Minimum und steigt in der praefebrilen Periode.

Betrachtet man die Ausscheidung im Vergleich zu Stickstoff, so gestaltet sie sich etwas anders, aber wesentliche Unterschiede sind nicht zu finden. So hat das Chlor das Maximum in der Fieberhöhe, das Minimum in der Apyrexie, Phosphor das Maximum in der praefebrilen Periode, das Minimum in der postfebrilen, Calcium das Minimum in der Apyrexie, das Maximum in der praefebrilen Zeit. Kalium das Minimum auf der Fieberhöhe, das Maximum in der postfebrilen Zeit. - Natrium erreicht das Minimum in der Apyrexie, das Maximum in der praefebrilen Zeit. Alloxurbasen werden in der praefebrilen Zeit am reichlichsten, in der Apyrexie am sparsamsten ausgeschieden. Ammoniak gerade umgekehrt.

Das Ansteigen der Temperatur ist mit einer Vermehrung des Chlors und Ammoniaks und einer Verminderung aller anderen Bestandtheile verbunden. Das Fallen der Temperatur bringt mit sich eine Vermehrung des Phosphors und Stickstoffs und Verminderung aller anderen Bestandtheile. Calcium, Natrium und Kalium haben erst in der zweiten Periode des Fiebers eine Vermehrung gezeigt.

Die Untersuchung der Ausscheidung bei den verschiedenen Fiebertypen hat nur eines mit ziemlicher Sicherheit ergeben: Die Ausscheidung bei Fieberabnahme kann die Merkmale einer Fieberausscheidung verstärkt zeigen. Die Ausscheidung bei Fiebererhöhung kann dieselben Merkmale schwächer zu Tage treten lassen.

Wenn ich hier von Fieberausscheidung schlechthin spreche, so verstehe ich darunter die bekannte Retention von Chlor und die Vermehrung des Phosphors und Stickstoffs. Dieses kann nun auch bei Fieberabnahme auftreten und kann bei Fiebererhöhung verschwinden. 
Näher beschrieben bedeutet dieses, dass nach dem Abfall der Temperatur das Chlor erst recht zurïckgehalten wird und der Stickstoff and Phosphor erst recht ausgeschieden werden. Folgt dann eine nochmalige Erhöhung der Temperatur, so wird das Chlor vermehrt, obgleich es retinirt sein sollte, der Phosphor wird im geringeren Maasse ausgeschieden, statt vermehrt zu sein.

Es scheint, dass der Organismuss anders bei dem Anfange und Ende des Fiebers reagire als bei dessen Dauer. Das Fieber bringt auf die Dauer die bekannten Veränderungen mit sich; wird aber auch während der Dauer des Fiebers die Temperatur erhöht, so entsteht ein Bild, welches der Genesung entspricht (der fieberlosen Periode). Entsteht eine Verminderung der Körperwärme, so tritt das verschärfte Bild des Fiebers auf. Vermehrung und Verminderung der Temperatur sollen hier als Maass des Fiebers dienen. Die Frage, inwiefern sie dazu berechtigt sind, wollen wir hier nicht berühren.

Wenn wir uns also nach dem Gesagten ein Schema des Fiebers entwerfen wollten, so hätte dieses folgende Gestalt: Die erste Erhöhung der Temperatur soll eine Vermehrung des Chlors, [Natrium, Kalium, Calcium, Ammoniak lasse ich aus, um das Bild nicht zu compliciren], eine Verminderung des Stickstoffs (oder Vermehrang), eine Verminderung des Phosphors nach sich ziehen. - Die zweite Periode, wenn das Fieber unverändert andauert, zeigt ein allmähliches Sinken der Chlorausscheidung, ein Steigen des Stickstoffs (oder Verbleiben auf der früheren Höhe) ein Sinken des Phosphors. - Die dritte Periode - immer bei gleicher Temperatur - zeigt ein Sinken des Chlors, ein Steigen des Phosphors und des Stickstoffs, und zwar ist jetzt das Chlor unter Norm gesunken, Phosphor und Stickstoff über Norm gestiegen. - Jetzt haben wir den Ausscheidungstypus, der als Fieberharn bekannt ist. - Dieses Bild kann nun andauern, wenn die Temperatur unverändert bleibt. Fällt die Temperatur (vielleicht durch Verdünnung des Blutes), so tritt das vierte Stadium ein, das Chlor wird noch mehr retinirt, der Phosphor und der Stickstoff noch mehr ausgeschieden. Dauert das Fallen der Temperatur, so erreicht Chlor das Minimum, Phosphor und Stickstoff ihr Maximum, und es ändert sich das Bild allmählich. Es tritt das fünfte Stadium ein, die Chloraus- 
scheidung steigt bis zur Norm, Phosphor and Stickstoff kommen zur normalen Menge zurück.

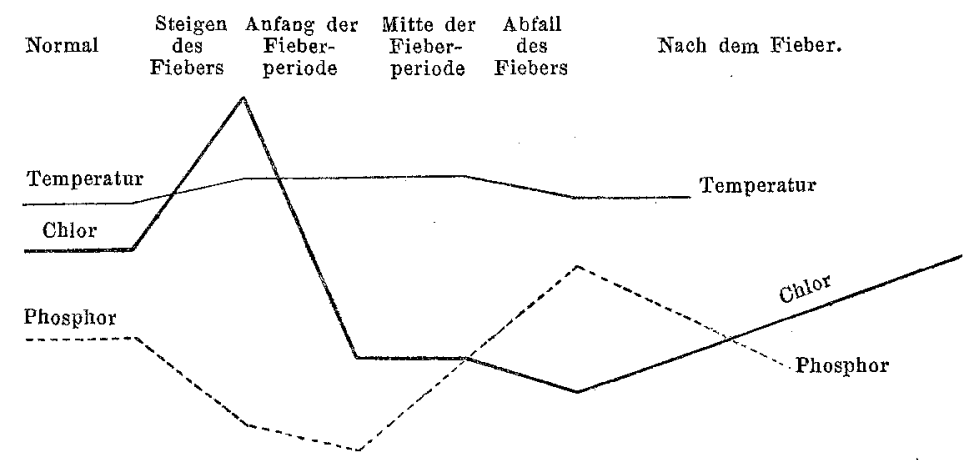

I. Periode. II. Per. IIr. Per. IV. Per. V. Per.

vi. Periode.

Dieses wäre das Bild des Fiebers, wie es bei Malaria auftritt und wie wir es bei den anderen Fieberformen gefunden haben.

Sollte man sich an eine Erklärung wagen, so muss vorausgesetzt werden, dass die ausführenden Gefässe es sind, oder vielmehr das Blut es ist, welches die Veränderungen im Harne mitmacht. Das Blut ist wasser- und chlorreicher als die Gewebe, dagegen phosphorund stickstoffärmer.

Wenn wir also am Anfang des Fiebers (I. Periode) den Harn chlorreich und phosphorarm finden, so bedeutet dieses, dass der Strom von den Geweben in das Blut unterbrochen ist, das Blut giebt den Geweben wenig Wasser, wenig Chlornatrium abnimmt wenig Phosphor und Stickstoff auf. - Es entspricht dieses einer Circulation, welche entweder durch Verengerung der Gefässe, oder durch Verlangsamrung des Blutstromes den Diffusionsstrom wenig ausgiebig gemacht hat.

Diese Art der Circulation dauert nicht lange, es folgt ihr ein Stadium der Erweiterung der Gefässe und rascher Circulation, und jetzt tritt das II. und III. Stadium auf. - Das wasserreichere Blut giebt sein Wasser und Kochsalz den Organen, retinirt das von anssen zugefügte Wasser und nimmt die stickstoffhaltigen Stoffe mit sich. Die Zersetzung der Stickstoffbestandtheile geht rasch vor sich, die phosphorhaltigen sind beständiger, deshalb tritt das Minimum des Phosphors jetzt auf und deshalb steigt der Stickstoff im Harn rascher als der Phos- 
phor. So stehen wir auf der Höhe des Fiebers und das zerfallende grosse Eiweissmolekül liefert immer mehr Stoffe, deren kleines Molekül den osmotischen Druck vergrössert und immer mehr Wasser und Salze retinirt: $)$. Der Eiweisszerfall wird somit durch $W$ ässerigwerden des Organismus bedingt und unterhalten. - Wenn jetzt das Fieber zu fallen beginnt, wenn also die Circulation besser wird und die Organe das Wasser abgeben, so wird das Bild des Fiebers verschärft; denn der Strom kehrt sich um, und statt vom Blut zu den Organen geht jetzt von den Organen in's Blut das Wasser mit seinen Salzen, welche chlorarm und phosphorreich sind. Die Ausscheidung des Wassers wird vermehrt, nicht aber die des Kochsalzes; mit dem Wasser treten Phosphorsalze und Stickstoff aus, bis sich das Gleichgewicht herzastellen beginnt und alles zur Norm zurückkehrt. Stellen wir uns während des Stadiums des Abfallens des Fiebers eine plötzliche Erhöhung des Fiebers vor, so wird durch die Circulationsstörung die Phosphor-Ausscheidung verhindert, Chlor vermehrt; denken wir uns während des Fiebers ein Sinken der Temperatur, so tritt das Umgekehrte auf.

Aus den Untersuchungen von Rosenthal ${ }^{1}$ ), E. Arousohn ${ }^{2}$ ), Geigel ${ }^{3}$ ), Maragliano ${ }^{4}$ ) geht hervor, dass eine Verminderung der Wärmeabgabe (eine Verengerung der Gefässe) bei acut Fiebernden stattfindet, wenn die Temperatur ansteigt ( $R_{0}$ s enthal), dass nach dem Temperaturabfall und während desselben das Volumen der Gefässe ansteigt (Pletismograph - Versuche von Maragliano).

Die vermehrte Stickstoff-Ausscheidung, im Stadium der latenten Fieberperiode von Naunyn beobachtet, soll dem Gesagten nicht widersprechen; denn $1^{0}$ ist auch bei unseren Beobachtungen oft ein Ansteigen des $\mathrm{N}$ mit der Temperatur beobachtet, $2^{\circ}$ betreffen die Versuche Naunyns Hunde, welchen subcutane Jauche ein-

*) Vergleiche darüber E. Schulze, Zeitschr. für phys. Chemie. Bd. XXIII. S. 18. Ueber den Umsatz der Eiweisstoffe in der lebenden Pflanze.

1) Rosenthal, Archiv für Anath. and Phys. Phys. Abth. S. 1. 1888.

${ }^{2}$ ) Aronsohn, Deutsche Med. Wochenscbr. 1888. No. 3, 4.

3) Geigel, Verhandlungen der Phys. Med. Gesellschaft in Würzburg. Bd. XXII. S. 1.

4) Maragliano, Riforma Med. 1888. Archiv ital. de Biologi. XI. p. 195, 
gespritzt wurde, somit das Fieber mit dem Zerfall im Blute begann, wie auch bei Malaria dieses der Fall ist.

Die Röhmann'sche Erklärung der Chlor-Retention durch das Gewebe-Eiweis kann neben dem Gesagten bestehen. Es wurde von mir eine ähnliche Meinung ausgesprochen bei Anlass der Untersuchung des Stoffwechsels bei Anaemien; ich glaube aber, dass auch ohne diese Annahme das Chlor Grund genug hat, retinirt zu werden.

Die vermehrte Chlor-Ausscheidung wurde von v. Terray auf den Zerfall der Blatkörperchen zurückgeführt. Die Blatkörperchen haben so wenig Chlor, dass sie wohl sämmtlich zerfallen müssten, um die nöthige Menge zu liefern. Wenn aber ein chronischer Blatkörperchen-Zerfall stattfindet, so ist das Chor durchaus nicht vermehrt, sondern im Gegentheil vermindert, wie dieses bei allen chronischen Anaemien stattfindet.

Auch über die postfebrile N-Ausscheidung exestiren Theorien. So glaubt man mit Naunyn, dass die vermehrte N-Ausscheidung auf eine Anhäufung des $\mathrm{N}$ im Organismus zurückzuführen sei. Die mit Zerfallproducten überladenen Nieren geben auf einmal die ganze Ladung ab. Es wäre die Meinung schwer za widerlegen, wenn in der postfebrilen Periode neben der Anhäufung von $\mathrm{N}$ nicht eine Verminderung der Chlorausscheidung zu constatiren wäre. Dieses lässt uns glauben, dass eine Aenderung in der Circulation die Schuld aller der Phenomene ist. Es muss nach dem Fieber der Austausch zwischen den Geweben und den Blutgefässen ein reger oder ausgiebiger werden, und auf diese Weise kommt es zur Ausscheidung, welche ein verschärftes Bild der Fieber-Ausscheidung darstellt.

Diese postfebrile und praefebrile Gefässänderung übt besonders Wirkung aus auf lösliche Salze; denn nur die Natrium- und Kaliumsalze - wohl als lösliche Chloride - zeigen die Eigenthümlichkeit des raschen Absteigens und ebenso raschen Fallens. Das Calcium-Phosphat folgt nicht so rasch den Schwankungen und bleibt bei dem typischen Verhalten: fällt mit dem Steigen der Temperatur, steigt mit dem Fallen. - Auch der Phosphor und die Ammoniumsalze gehören $z u$ den trägen Substanzen. Der Phosphor-Ausscheidung muss der Nucleinzerfall vorausgehen, deshalb erreicht Phosphor erst im zweiten Stadium sein Maximum 
und überdauert das Fieber. Auch die Bildung der Ammoniumsalze bedarf eines "Sanerwerdens" ${ }^{\text {I }}$ ) der Säfte, deshalb der Parallelismus zwischen Amoniak und Phosphor.

Das merkwürdigste Verhalten zeigt ohne Zweifel das Kalium, und wenn wir die vermehrte Ausscheidung bei Steigen der Temperatur mit der raschen Diffusion erklären, so fehlt uns jede Erklärung für das Sinken in der postfebrilen Zeit (welches übrigens keine Regel bildet, ausser wir müssten annehmen, dass es hier den gleichen Gesetzen folge, wie das Natrium.

Die Erklärung, mag sie geschickt oder ungeschickt sein, bildet nicht das $W$ esentliche und ändert nichts an der Beobachtung. In den alten Arbeiten von Langley und Huppert haben wir Zahlen gefunden, welche im Lichte unserer Erklärung an Bedeutung gewinnen, dort unbeachtet oder minderwerthig angesehen waren. So möchten auch wir durch unsere Beobachtung für andere Theorien nur ein zuverlässiges Material geliefert haben wollen.

1) "Sauerwerden“ im Sinne des früher Gesagten durch Verminderung der Alkalescenz, welche Verminderung mit der Vermebrung des Ammoniaks zusammenfällt. 